\title{
Conformal Mapping of Long Quadrilaterals and Thick Doubly Connected Domains
}

\author{
R. Laugesen
}

\begin{abstract}
In this paper we investigate theoretically an approximation technique for avoiding the crowding phenomenon in numerical conformal mapping. The method applies to conformal maps from rectangles to "long quadrilaterals," i.e., Jordan domains bounded by two parallel straight lines and two Jordan arcs, where the two ares are far apart. We require that these maps take the four corners of the rectangle to the four corners of the quadrilateral.

Our main theorem tackles a conformal mapping problem for doubly connected domains, and we derive from this our results for quadrilaterals. As a corollary, we extend the "domain decomposition" mapping technique of Papamichael and Stylianopoulos.

Similar results hold for the inverse maps, from quadrilaterals to rectangles.
\end{abstract}

\section{Introduction}

When one tries to compute numerically a conformal map of an elongated region, one is frequently obstructed by the "crowding" phenomenon: points that are uniformly distributed in the domain can come exponentially close to one another in the image, and vice versa. A more helpful feature of mappings of elongated regions is that they are often "localized": an alteration of the boundary at one end of the region will not change the mapping near the other end much. In this paper we present a method that both avoids crowding and takes advantage of localization for the conformal mapping of a particular class of long quadrilaterals, shown in Fig. 1. We now describe our main results.

A quadrilateral is a Jordan domain $G$ in the complex plane together with four distinct points $a, b, c, d$ of $\partial G$, listed in counterclockwise order. Given such a quadrilateral $G$, a unique $h>0$ and a unique conformal map $g$ onto $G$ from the rectangle $R=(-h / 2, h / 2) \times(0,1)$ exist such that $g$ takes the four corners $h / 2$, $h / 2+i,-h / 2+i,-h / 2$ of $R$ onto the points $a, b, c, d$, respectively. The length $h$ of $R$ is called the conformal module of the quadrilateral $G$.

Suppose $G$ has the form shown in Fig. 1. That is, the Jordan curve $\partial G$ intersects $\{w: \operatorname{Im} w=0\}$ in the segment $[d, a]$, intersects $\{w: \operatorname{Im} w=1\}$ in the segment $[c, b]$,

Date received: April 29, 1993. Date revised: July 30, 1993. Communicated by Dieter Gaier. AMS classification: Primary 30C35; Secondary 65E05, 30E10.

Key words and phrases: Conformal mapping, Quadrilaterals, Approximation. 


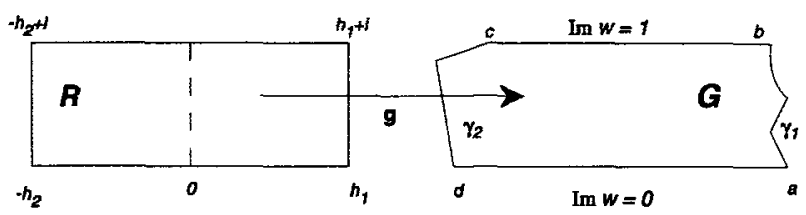

Fig. 1. The conformal map $g$ of the rectangle $R$ onto the quadrilateral $G$.

and intersects $\{w: 0<\operatorname{Im} w<1\}$ in the Jordan arcs $\gamma_{1}$ and $\gamma_{2}$. Throughout this introduction we take $h_{1}=h / 2=h_{2}$ in Fig. 1. Write $R_{1}$ for the half-strip $(-\infty, h / 2) \times(0,1)$ and write $G_{1}$ for the Jordan domain bounded by

$$
[-\infty, a] \cup \gamma_{1} \cup[-\infty+i, b] .
$$

Let $g_{1}$ be the conformal map from $R_{1}$ onto $G_{1}$ that takes $h / 2, h / 2+i, \infty$ to $a, b$, $\infty$, respectively, as in Fig. 2 (with $h_{1}=h / 2$ ).

We will show that $g$ is uniformly well approximated by $g_{1}$ on the right-hand half of $R$ provided $G$ is long (in the sense that its conformal module $h$ is large) and provided $\gamma_{1}$ is "nice." In particular, Theorem 4 and Corollary 2 below give the following. Suppose that $\alpha \in(0,1]$ and that $\gamma_{1}$ can be parametrized as $\gamma_{1}(y)=$ $x(y)+i y, y \in[0,1]$, where $x(y)$ is absolutely continuous and

$$
\underset{0<y<1}{\operatorname{ess} \sup }\left|x^{\prime}(y)\right| \leq \cot \left(\frac{\pi \alpha}{2}\right) \text {. }
$$

Then

$$
\left|g(z)-g_{1}(z)\right| \leq M^{\prime} e^{-\pi h \min (\alpha, 1 / 2)}, \quad z \in R, \quad \operatorname{Re} z>0,
$$

where $M^{\prime}>0$ depends only on $\gamma_{1}$. We construct an example to show that the order of approximation $O\left(e^{-\pi h \min (\alpha, 1 / 2)}\right)$ in (1.2) is sharp as $h \rightarrow \infty$. Clearly, this order of approximation is never better than $O\left(e^{-\pi h / 2}\right)$, but it can be worse when $\alpha<\frac{1}{2}$. These results have obvious analogues for the left-hand half of $R$, in which $\operatorname{Re} z<0$.

The reason we search in this paper for a good approximation to $g$ is that when $G$ is even moderately long, say $h>10$, the standard numerical procedure for approximating $g$ can break down due to the "crowding effect." The standard procedure is first to map $R$ to a disk and then to map that disk to G. Roughly

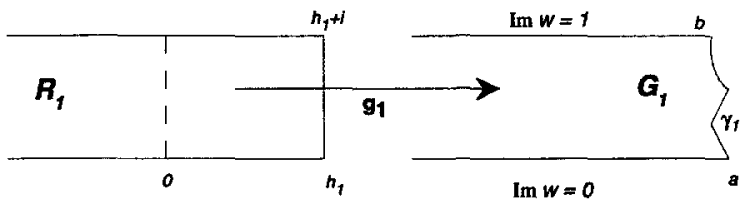

Fig. 2. The conformal map $g_{1}$ of the half-strip $R_{1}$ onto $G_{1}$. 
speaking, the intermediate images of $h / 2, h / 2+i$ (respectively $-h / 2+i,-h / 2$ ) on the boundary of the disk are only about $e^{-\pi h / 2}$ apart, yet they must subsequently be mapped to the points $a, b$ (respectively $c, d$ ) at distance $\geq 1$ from each other on the boundary of $G$. This exponential crowding of points on the boundary of the disk can cause serious numerical problems, and can be thought of as a kind of ill-conditioning of the composite map from $R$ to $G$.

For more detailed accounts of the crowding effect and its impact on numerical conformal mapping, see [PaKW, Section 2], [Pa, pp. 68, 80], [PaS4], [We1], [We2], [MeZ], and [PaS2, p. 353]. For previous work on long quadrilaterals, see [GaP], [MoS], [PaKW], [PaS2], and [SeK]. Howell and Trefethen show how to eliminate crowding for long polygons in [HoT]; their idea is to avoid the intermediate mapping to a disk by defining a "Schwarz-Christoffel" transformation directly on the long rectangle. Also see [DeLE], where an ellipse rather than a disk is used as the standard domain for conformal mapping.

One reason that $g_{1}$ is a worthwhile approximation to $g$ is that $g_{1}$ can be evaluated numerically in a way that avoids crowding. Indeed, $z \mapsto e^{\pi z}$ takes $R_{1}$ onto the upper half of the disk $\Delta\left(0, e^{\pi h / 2}\right)$, while $w \mapsto e^{\pi w}$ takes $G_{1}$ conformally onto a Jordan domain $D_{1}^{\prime}$ in the upper half-plane whose boundary intersects the real axis in the segment $\left[e^{\pi b}, e^{\pi a}\right]$. Let $D_{1}$ be the Jordan domain formed by the union of $D_{1}^{\prime}$ and its reflection in the real axis, together with $\left[e^{\pi b}, e^{\pi a}\right]$. Let $f_{1}: \Delta\left(0, e^{n h / 2}\right) \rightarrow D_{1}$ be the conformal map with $f_{1}(0)=0$ that is positive on the positive real axis. Clearly,

$$
g_{1}(z)=\frac{1}{\pi} \log f_{1}\left(e^{\pi z}\right), \quad z \in R_{1}
$$

where " $\log$ " denotes the branch of the logarithm with $-\pi<\operatorname{Im} \log \leq \pi$. Thus to evaluate $g_{1}$ it is enough to evaluate $f_{1}$. This can be done numerically without undue fear of crowding since $D_{1}$ is not elongated, in general.

Another feature of our method is that it takes advantage of the localized nature of $g$ : in constructing $g_{1}$ we simply throw away the left-hand boundary arc $\gamma_{2}$ of $\partial G$, but still we find that $g_{1}$ gives a good approximation to $g$ in the right-hand half of $R$. Thus, in general terms, it is the localization of $g$ that enables us to reduce the problem to that of computing $g_{1}$ and hence to the well-studied problem of computing the Riemann map $f_{1}$.

Papamichael and Stylianopoulos [PaS2] have also approximated $g$ in a way that avoids crowding, by using the "exponentiation then reflection" trick described above together with a domain decomposition idea and Garrick's integral equation method. They assume $\alpha>\frac{1}{2}$ in (1.1), so that ess $\sup _{y}\left|x^{\prime}(y)\right|<1$, and then they approximate $g$ by the conformal map $q_{1}$ from the right-hand half of $R$ onto the right-hand "hall" of $G$. We shall see that their result

$$
g-q_{1}=O\left(e^{-\pi h / 2}\right)
$$

follows from the " $\alpha>\frac{1}{2}$ " case of (1.2). In Theorem 4 and Corollary 3 we generalize their result to arbitrary $\alpha \in(0,1]$. Papamichael and Stylianopoulos [PaS1, Con- 
jecture 3.1] conjectured on the basis of experimental evidence that (1.3) holds for all $\alpha$, but we disprove this for $\alpha<\frac{1}{2}$, by means of an example in Section 5 .

Incidentally, our approximation $g_{1}$ is generally easier to compute numerically than the map $q_{1}$ used by Papamichael and Stylianopoulos: $g_{1}$ is computed from the map $f_{1}$ that goes from a disk to a simply connected domain, whereas $q_{1}$ is computed in the corresponding way from a map that goes from an annulus to a doubly connected domain.

Our final results address the "inverse" problem of approximating $g^{-1}$ by $g_{1}^{-1}$ and $q_{1}^{-1}$. Let

$$
\sigma=\min _{w \in \gamma_{1}} \operatorname{Re} w-\max _{w \in \gamma_{2}} \operatorname{Re} w
$$

In Corollaries 6 and 7 we show that

$$
g^{-1}-g_{1}^{-1}=O\left(e^{-\pi \sigma / 2}\right) \text { and } g^{-1}-q_{1}^{-1}=O\left(e^{-\pi \sigma / 2}\right)
$$

uniformly in the right-hand "half" of $G$, with no assumptions on $\gamma_{1}$ or $\gamma_{2}$. If $\sigma \geq 10$, for example, these error terms $O\left(e^{-\pi \sigma / 2}\right)$ are at most $10^{-6}$ or so.

\section{Statement and Discussion of Results}

Our main theorem, Theorem 1, deals with approximating the conformal map onto a doubly connected domain. In Corollary 2 we derive from this an approximation to the conformal map onto a quadrilateral. Corollary 3 gives a similar result for the domain decomposition approach. In Theorem 4 we assume that $\gamma_{1}$ has bounded slope and then deduce that our previous results apply. This enables us to generalize the results of Papamichael and Stylianopoulos and to answer in the negative two questions raised by them. Lastly, we estimate the errors for the approximations in the inverse directions, in Theorem 5 and Corollaries 6 and 7.

For Theorem 1 we consider a doubly connected domain $D$ in the Riemann sphere that is symmetric in the real axis. Let the components of $D^{c}$ be $K_{1}$ and $K_{2}$, and assume that $\infty \in K_{1}$ and $0 \in K_{2}$. Let $0<r_{2}<1<r_{1}<\infty$ and suppose that

$$
f:\left\{r_{2}<|z|<r_{1}\right\} \rightarrow D
$$

is a conformal map that is symmetric in the real axis and is positive on the positive real axis. Suppose further that the inner complementary components $\left\{z:|z| \leq r_{2}\right\}$ and $K_{2}$ correspond under $f$. See Fig. 3. Let $D_{1}=C \backslash K_{1}$ and take

$$
f_{1}:\left\{|z|<r_{1}\right\} \rightarrow D_{1}
$$

to be the conformal map with $f_{1}(0)=0$ and $\left(f_{1}^{-1} \circ f\right)\left(r_{1}\right)=r_{1}$, as in Fig. 4 . Note that $f_{1}$ also is symmetric in the real axis. Define

$$
\left\|f_{1}\right\|_{\min }=\liminf _{|z| \rightarrow r_{1}}\left|f_{1}(z)\right|=\min _{w \in \partial D_{1}}|w|=\min _{w \in K_{1}}|w| .
$$




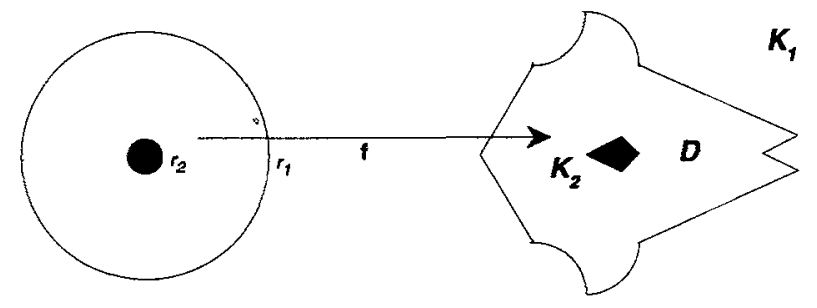

Fig. 3. The conformal map $f$ onto the doubly connected domain $D$.

Write

$$
r=\frac{r_{2}}{r_{1}} \quad \text { and } \quad r_{*}=\max \left(r_{2}, \frac{1}{r_{1}}\right)
$$

so that $r_{*}<1$ and $r \leq r_{*}^{2}$. When $r_{*}$ is small, we say $D$ is a thick doubly connected domain.

The next theorem shows that $\log f_{1}$ provides a good approximation to $\log f$ for $|z| \geq 1$, as long as $D$ is thick and $f_{1}$ is a Hölder map. We prove it in Section 3.

Theorem 1. Suppose $f_{1}$ is a Hölder map of order $\alpha \in(0,1]$, with

$$
\left|f_{1}(z)-f_{1}(w)\right| \leq M\left\|f_{1}\right\|_{\min } r_{1}^{-\alpha}|z-w|^{\alpha}, \quad|z|,|w|<r_{1},
$$

for some $M>0$. Then $r_{0}>0$ exists such that if $r_{*} \leq r_{0}$, then

$$
\left|\log f(z)-\log f_{1}(z)\right| \leq \max \left(10 r_{2}, \frac{2}{\sqrt{3}} 4^{\alpha} M r^{\alpha}\right)=O\left(r_{*}^{\min (1,2 \alpha)}\right), \quad 1 \leq|z|<r_{1} .
$$

Here $r_{0}$ depends only on $\alpha$ and $M$, and we can take

$$
r_{0}=\min \left(e^{-\pi},\left(4^{\alpha} M\right)^{-1 / 2 \alpha}\right) .
$$

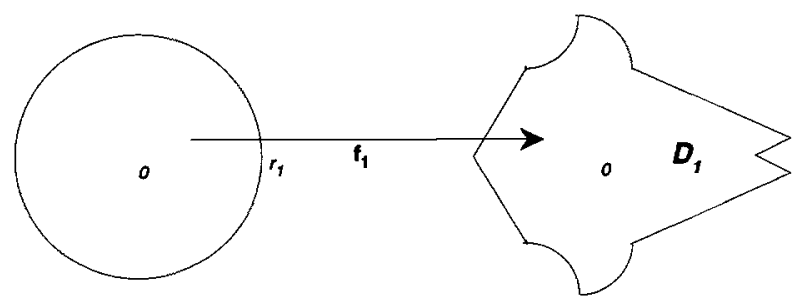

Fig. 4. The conformal map $f_{1}$ onto the simply connected domain $D_{1}$. 
Actually, the hypotheses of the theorem imply a more precise, pointwise estimate, which we also prove in Section 3:

$$
\left|\log f(z)-\log f_{1}(z)\right| \leq\left[10 r_{2}\right]^{1-\log |z| / \log r_{1}}\left[\frac{2}{\sqrt{3}} 4^{\alpha} M r^{\alpha}\right]^{\log |z| / \log r_{1}}, \quad 1 \leq|z|<r_{1} .
$$

Note that this estimate simplifies to an expression like (2.2) when $|z|=1$ and as $|z| \rightarrow r_{1}$; that is, the order of approximation in (2.4) is like $O\left(r_{2}\right) \leq O\left(r_{*}\right)$ when $|z|=1$, and becomes like $O\left(r^{\alpha}\right) \leq O\left(r_{*}^{2 \alpha}\right)$ as $|z| \rightarrow r_{1}$.

A few remarks on the theorem are in order. First, we use in the theorem the branch of the logarithm having $-\pi<\operatorname{Im} \log \leq \pi$. Second, it is indeed reasonable to write " $O\left(r_{*}^{\min (1,2 \alpha)}\right)$ " in (2.2), because, for each small $r_{*}$, there is an " $f_{1}$ " that is Hölder of order $\alpha$ with the very same Hölder constant $M$. To see this, take $\lambda>1$ to be large and replace $K_{1}$ and $K_{2}$ by their dilates $\lambda K_{1}$ and $K_{2} / \lambda$. It is found that the map " $f_{1}$ " for the domain $\mathbf{C} \backslash\left(\lambda K_{1}\right)$ is Hölder of order $\alpha$ with Hölder constant $M$, while the corresponding " $r_{*}$ " is small.

Third, it must be admitted that the theorem invites the question: "If all we are given is the domain $D$, then how are we to know $f_{1}$ is Hölder?" We address this question in Theorem 4 and its associated discussion.

Fourth, the assumption that $D, f$, and $f_{1}$ are all symmetric in the real axis is not of vital importance-it simply leads to better constants in the theorem.

Finally, in Section 5 we show that the order of approximation $O\left(r_{*}^{\min (1,2 \alpha)}\right)$ in Theorem 1 is sharp in the following sense. Fix $\alpha \in(0,1]$. For each small $r>0$ we construct a domain $D$ such that (with $r_{2}=r^{1 / 2}, r_{1}=1 / r^{1 / 2}, r_{*}=r^{1 / 2}$ ) the map $f_{1}$ is Hölder of order $\alpha$ and satisfies (2.1) with Hölder constant $M$, but

$$
\sup _{1 \leq|z|<r_{1}}\left|\log f(z)-\log f_{1}(z)\right| \geq M^{\prime \prime} r_{*}^{\min (1,2 \alpha)} \text {. }
$$

Here the constants $M$ and $M^{\prime \prime}$ depend only on $\alpha$, and so letting $r \rightarrow 0$ shows that the order of approximation $O\left(r_{*}^{\min (1,2 \alpha)}\right)$ is best possible.

Next we use Theorem 1 to obtain approximations to the conformal map from a rectangle onto a quadrilateral. Let $G$ be a quadrilateral of the form shown in Fig. 1, so that $\partial G$ intersects $\{\operatorname{Im} w=0\}$ in $[d, a]$ and intersects $\{\operatorname{Im} w=1\}$ in $[b, c]$. Let $h$ be the conformal module of $G$ and let $h_{1}$ and $h_{2}$ be positive numbers with $h=h_{1}+h_{2}$. For increased generality we redefine the rectangle $R$ to be

$$
R=\left(-h_{2}, h_{1}\right) \times(0,1)
$$

recall that in the introduction we simply took $h_{1}=h / 2=h_{2}$. Write $g$ for the conformal map of $R$ onto $G$ that takes the four corners $h_{1}, h_{1}+i,-h_{2}+i,-h_{2}$ of $R$ onto $a, b, c, d$, respectively. Now let $G_{1}$ be the Jordan domain bounded by $[-\infty, a] \cup \gamma_{1} \cup[-\infty+i, b]$, as in Fig. 2, and redefine the half-strip $R_{1}$ to be

$$
R_{1}=\left(-\infty, h_{1}\right) \times(0,1) .
$$

Let $g_{1}$ be the conformal map from $R_{1}$ onto $G_{1}$ that takes $h / 2, h / 2+i, \infty$ to $a, b$, $\infty$, respectively, as in Fig. 2. 
We now place ourselves in the situation covered by Theorem 1. Put

$$
D^{\prime}=e^{\pi G},
$$

and let $D$ be the union of $D^{\prime}$ and its reflection in the real axis, together with the intervals $\left[e^{\pi b}, e^{\pi c}\right]$ and $\left[e^{\pi d}, e^{\pi a}\right]$. Thus $D$ is a doubly connected domain that is symmetric in the real axis. Let

$$
r_{2}=e^{-\pi h_{2}}, \quad r_{1}=e^{\pi h_{1}} .
$$

We can define a conformal map $f:\left\{r_{2}<|z|<r_{1}\right\} \rightarrow D$ by putting

$$
f(z)=e^{\pi g((1 / \pi) \log z)}
$$

when $\operatorname{Im} z \geq 0$, and putting $f(z)=\overline{f(\bar{z})}$ when $\operatorname{Im} z<0$; here "log" is the branch of the logarithm with $-\pi<\operatorname{Im} \log \leq \pi$.

Now put $D_{1}^{\prime}=e^{\pi G_{1}}$ and let $D_{1}$ be the union of $D_{1}^{\prime}$ and its reflection in the real axis, together with the interval $\left[e^{\pi b}, e^{\pi a}\right]$. Thus $D$ is a Jordan domain that is symmetric in the real axis. We define a conformal map $f_{1}:\left\{|z|<r_{1}\right\} \rightarrow D_{1}$ by putting

$$
f_{1}(z)=e^{\pi g_{1}((1 / \pi) \log z)}
$$

when $\operatorname{Im} z \geq 0$ (with $f_{1}(0)=0$ ), and putting $f_{1}(z)=\overline{f_{1}(\bar{z})}$ when $\operatorname{Im} z<0$. Note that $\left(f_{1}^{-1} \circ f\right)\left(r_{1}\right)=r_{1}$, and that all the other preliminary requirements of Theorem 1 are satisfied by $f$ and $f_{1}$.

From Theorem 1 we immediately deduce that $g$ is well approximated by $g_{1}$ provided $f_{1}$ is Hölder and

$$
h_{*}=\min \left(h_{1}, h_{2}\right)
$$

is large. Note that $r_{*}=e^{-\pi h_{*}}$ and $h \geq 2 h_{*}$.

Corollary 2. Suppose $f_{1}$ is a Hölder map of order $\alpha \in(0,1]$, with

$$
\left|f_{1}(z)-f_{1}(w)\right| \leq M\left\|f_{1}\right\|_{\min } r_{1}^{-\alpha}|z-w|^{\alpha}, \quad|z|,|w|<r_{1},
$$

for some $M>0$. Then $h_{0}>0$ exists such that if $h_{*} \geq h_{0}$, then

$$
\left|g(z)-g_{1}(z)\right| \leq \frac{1}{\pi} \max \left(10 e^{-\pi h_{2}}, \frac{2}{\sqrt{3}} 4^{\alpha} M e^{-\pi h \alpha}\right)=O\left(e^{-\pi h_{*} \min (1,2 \alpha)}\right)
$$

for all $z \in R$ with $\operatorname{Re} z>0$.

In view of (2.3), we may take

$$
h_{0}=\max \left(1, \frac{1}{2 \pi \alpha} \log \left(4^{\alpha} M\right)\right) .
$$

In Section 5 we show that the order of approximation $O\left(e^{-\pi h_{*} \min (1,2 \alpha)}\right)$ in the corollary is best possible. 
Note also that from (2.4) we derive the pointwise estimate

$$
\left|g(z)-g_{1}(z)\right| \leq \frac{1}{\pi}\left[10 e^{-\pi h_{2}}\right]^{1-\operatorname{Re} z / h_{1}}\left[\frac{2}{\sqrt{3}} 4^{\alpha} M e^{-\pi h \alpha}\right]^{\operatorname{Re} z / h_{1}}, \quad z \in R, \quad \operatorname{Re} z>0
$$

provided $h_{*} \geq h_{0}$

In the special case that $\gamma_{2}$ is a vertical line segment, we obtain a much stronger result than Corollary 2 by reflecting $G$ in $\gamma_{2}$ to obtain a new quadrilateral that is "twice as long." Indeed, by applying Corollary 2 to this new quadrilateral we find that $g-g_{1}=O\left(e^{-\pi h \min (1,2 \alpha)}\right)$ uniformly in all of $R$.

If we wish to use Corollary 2 in practice, we must confront the fact that the domain of $g_{1}$ depends on the conformal module $h$ of $G$, and that we do not necessarily know $h$ if all we are given initially is the quadrilateral $G$. This difficulty can be circumvented by using an approximation $\tilde{h}$ to $h$ such as the one developed and applied in [PaS2]-[PaS4] by Papamichael and Stylianopoulos and extended in [GaH1] and [GaH2] by Gaier and Hayman; see also [La, Theorem 2], [MoS, p. 133], and [PaS2, Remark 5.3]. Specifically, given the approximation $\tilde{h}$, we put $h_{1}=\tilde{h} / 2$ and $h_{2}=h-\tilde{h} / 2$, so that $h_{1}+h_{2}=h$.

For the next corollary we decompose the quadrilateral $G$ into two simpler quadrilaterals $Q_{1}$ and $Q_{2}$, then approximate $g$ on the right-hand half of $R$ by a map onto $Q_{1}$. This is the domain decomposition approach of Papamichael and Stylianopoulos [PaS1], [PaS2].

Assume now that the right-hand arc $\gamma_{1}$ of $\partial G$, along with its endpoints, lies entirely in the right half-plane and that the left-hand arc $\gamma_{2}$ and its endpoints lie entirely in the left half-plane. Define quadrilaterals

$$
Q_{1}=G \cap\{w: \operatorname{Re} w>0\} \quad \text { and } \quad Q_{2}=G \cap\{w: \operatorname{Re} w<0\}
$$

with distinguished boundary points $a, b, i, 0$ and $0, i, c, d$, respectively, as in Fig. 5. Assume $h_{1}$ actually equals the conformal module of $Q_{1}$, so that a conformal map

$$
q_{1}: R \cap\{\operatorname{Re} z>0\} \rightarrow Q_{1}
$$

exists that takes the corners $h_{1}, h_{1}+i, i, 0$ to $a, b, i, 0$, respectively. Note that $h_{2}$ must be at least as large as the $\operatorname{module} \bmod \left(Q_{2}\right)$ of $Q_{2}$, since

$$
h_{1}+h_{2}=h=\bmod (G) \geq \bmod \left(Q_{1}\right)+\bmod \left(Q_{2}\right)=h_{1}+\bmod \left(Q_{2}\right)
$$

by Grötzsch's lemma [He, p. 437].

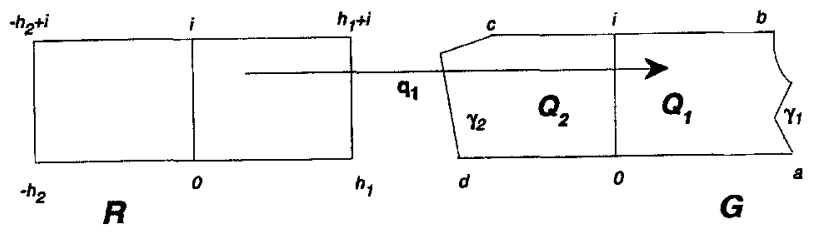

Fig. 5. Decompose $G$ into two smaller quadrilaterals $Q_{1}, Q_{2}$, then map the right-hand "half" of $R$ onto $Q_{1}$. 
The next corollary states that $q_{1}$ also serves as a good approximation to $g$ as long as $h_{*}$ is large and $f_{1}$ is Hölder. We prove it at the end of Section 3 and show in Section 5 that the order of approximation is again best possible.

Corollary 3. Suppose $f_{1}$ is a Hölder map of order $\alpha \in(0,1]$, with

$$
\left|f_{1}(z)-f_{1}(w)\right| \leq M\left\|f_{1}\right\|_{\min } r_{1}^{-\alpha}|z-w|^{\alpha}, \quad|z|,|w|<r_{1},
$$

for some $M>0$. Then $h_{0}>0$ exists such that if $h_{*} \geq h_{0}$, then

$$
\left|g(z)-q_{1}(z)\right| \leq \frac{2}{\pi} \max \left(10 e^{-\pi h_{*}}, \frac{2}{\sqrt{3}} 4^{\alpha} M e^{-2 \pi h_{*} \alpha}\right)=O\left(e^{-\pi h_{*} \min (1,2 \alpha)}\right)
$$

for all $z \in R$ with $\operatorname{Re} z>0$.

Again we may use

$$
h_{0}=\max \left(1, \frac{1}{2 \pi \alpha} \log \left(4^{\alpha} M\right)\right)
$$

In Section 3 we derive the pointwise estimate

$$
\left|g(z)-q_{1}(z)\right| \leq \frac{2}{\pi}\left[10 e^{-\pi h_{*}}\right]^{1-\operatorname{Re} z / h_{1}}\left[\frac{2}{\sqrt{3}} 4^{\alpha} M e^{-2 \pi h_{*}}\right]^{\operatorname{Re} z / h_{1}}, \quad z \in R, \quad \operatorname{Re} z>0,
$$

which is valid provided $h_{*} \geq h_{0}$. Notice that when $\operatorname{Re} z=0$, the right-hand side of (2.12) is $O\left(e^{-\pi h_{*}}\right)$, and when $\operatorname{Re} z=h_{1}$ it is $O\left(e^{-2 \pi h_{*} \alpha}\right)$. Thus if $\alpha>\frac{1}{2}$, the approximation $q_{1}$ is expected to be closer to $g$ on $\left\{z: \operatorname{Re} z=h_{1}\right\}$ than on $\{z: \operatorname{Re} z=0\}$. Papamichael and Stylianopoulos observed this occurring numerically in Remark 5.2 of [PaS2].

The point of the next theorem is that if the arc $\gamma_{1}$ of $\partial G$ is not too jagged, then $f_{1}$ is Hölder and so Corollaries 2 and 3 apply. We prove the theorem in Section 4.

Theorem 4. Let $\alpha \in(0,1]$. Suppose $\gamma_{1}$ can be parametrized as $\gamma_{1}(y)=x(y)+i y$, $y \in[0,1]$, where $x(y)$ is absolutely continuous and

$$
\underset{0<y<1}{\operatorname{ess} \sup }\left|x^{\prime}(y)\right| \leq \cot \left(\frac{\pi \alpha}{2}\right) .
$$

Then $f_{1}$ is Hölder of order $\alpha$, with

$$
\left|f_{1}(z)-f_{1}(w)\right| \leq M\left\|f_{1}\right\|_{\min } r_{1}^{-\alpha}|z-w|^{\alpha}, \quad|z|,|w|<r_{1},
$$

for some $M>0$. Thus Corollaries 2 and 3 apply, as well as the pointwise estimates (2.8) and (2.12). 
We may take

$$
M=4\left(1+\frac{2}{\alpha}\right) 3^{\alpha} e^{\pi \mathrm{Osc}\left(\gamma_{1}\right)} \max \left(1, \pi \mathrm{Osc}\left(\gamma_{1}\right)+\log 2\right)
$$

where

$$
\operatorname{Osc}\left(\gamma_{1}\right)=\max _{y} x(y)-\min _{y} x(y)
$$

measures the oscillation of $\gamma_{1}$ when it is regarded as a graph against the $y$ variable.

In Section 5 we show by example that the Hölder exponent $\alpha$ is best possible, in general.

The hypothesis (2.13) on the slope of $\gamma_{1}$ has an appealing geometric consequence: for each $y$, the open cone with vertex at $x(y)+i y$ and aperture $\pi \alpha$ that opens to the left lies entirely to the left of $\gamma_{1}$. Thus Theorem 4 is similar to the results of Lesley [Le] involving "interior $\alpha$-wedges." However, we prove our theorem directly so as to obtain explicit constants.

Many authors have considered the problem of finding conditions on the boundary that imply the Hölder continuity of $f_{1}$. Weaker conditions than (2.13) are certainly known: see [BeP], [Le], [NäP], [SmS] and the references in [Po2, p. 92]. We do not know, however, of any papers that give a Hölder constant $M$ depending explicitly just on $\alpha$ and on easily computable properties of $\gamma_{1}$. (Though Gaier did do this for the inverse map $f_{1}^{-1}$ in [Ga].)

Theorem 4 generalizes the result of Papamichael and Stylianopoulos [PaS2, (4.20)] that if (2.13) holds for $\alpha>\frac{1}{2}$, then the conclusions of Corollary 3 must hold, i.e.,

$$
g-q_{1}=O\left(e^{-\pi h_{*}}\right)
$$

Their conjecture [PaS1, Conjecture 3.1] that this estimate might remain valid for all $\alpha \in(0,1]$ is false for $\alpha<\frac{1}{2}$, as we show at the end of Section 5 by means of the same example we use to show Corollary 3 is sharp. We disprove Conjecture 3.2 of [PaS1] also.

\section{Numerical Usefulness of Theorem 4}

When the numerical examples studied by Papamichael and Stylianopoulos in [PaS1] and [PaS2] are examined, it is found that their estimates on $g-q_{1}$ are better than those given by combining Theorem 4 with Corollary 3 . One reason is that their examples are actually much "nicer" than the general case covered by Theorem 4. Specifically, in their examples the arcs $\gamma_{1}$ are Dini-smooth except perhaps for an inward-pointing corner, and so $f_{1}$ is actually Hölder of order 1 by a result due to $\mathrm{S}$. E. Warschawski, [Po2, Theorem 3.9]. In contrast, Theorem 4 can give only that $f_{1}$ is Hölder of some order $\alpha$ significantly less than 1 . Furthermore, the Hölder constants $M$ given by Theorem 4 are probably much larger than necessary for these examples. 


\section{Approximations in the Inverse Direction}

Our final three results concern the "inverse" approximation problem: How well do $g_{1}^{-1}$ and $q_{1}^{-1}$ approximate $g^{-1}$ in the right-hand "half" of $G$ ? Again we start with a theorem involving doubly connected domains and deduce from it corollaries about $g_{1}^{-1}$ and $q_{1}^{-1}$.

Consider again, as we did for Theorem 1, the doubly connected domain $D$, the simply connected domain $D_{1}$, the maps $f$ and $f_{1}$, and the numbers $r_{1}, r_{2}, r=r_{2} / r_{1}$. Recall that $D, D_{1}, f$, and $f_{1}$ are symmetric in the real axis. Assume in addition that the components $K_{1}$ and $K_{2}$ of $D^{c}$ lie in $\{w:|w|>1\}$ and $\{w:|w|<1\}$, respectively. From now on we measure the "thickness" of $D$ by means of the quantities

$$
\rho_{1}=\min _{w \in K_{1}}|w|>1, \quad \rho_{2}=\max _{w \in K_{2}}|w|<1, \quad \rho=\frac{\rho_{2}}{\rho_{1}}<1, \quad \rho_{*}=\max \left(\rho_{2}, \frac{1}{\rho_{1}}\right)<1 .
$$

Note that $\rho \geq r$ since $D$ is conformally equivalent via $f^{-1}$ to $\left\{z: r_{2}<|z|<r_{1}\right\}$. Put

$$
\kappa=\frac{1-e^{-1.5 \pi} / 2}{4} \approx \frac{1}{4}
$$

and define

$$
\beta(w)=\frac{\log \left(r_{1} /\left|f_{1}^{-1}(w)\right|\right)}{\log \left(\rho_{1} / \kappa\right)}
$$

for $w \in D$. Clearly, $\beta(w) \geq 0$.

The following theorem says that if $D$ is thick (in that $\rho_{*}$ is small), then $\log f_{1}^{-1}$ closely approximates $\log f^{-1}$ in the outer "half" of $D$. In the course of its proof we show that $0 \leq \beta(w) \leq 1$ when $|w|>1$.

Theorem 5. If $\rho_{*} \leq e^{-1.5 \pi}$, then

$$
\left|\log f^{-1}(w)-\log f_{1}^{-1}(w)\right| \leq\left[25 \rho_{2}\right]^{\beta(\omega)}[4.1 \rho]^{1-\beta(w)} \leq 25 \rho_{2}
$$

for all $w \in D$ with $|w|>1$.

Again we use the branch of the logarithm having $-\pi<\operatorname{Im} \log \leq \pi$.

In Section 6 we prove Theorem 5 and in Section 7 we show its order of approximation $O\left(\rho_{2}\right)$ is best possible. Note the main difference between the hypotheses of this theorem and those of Theorem 1: here we do not assume $f_{1}$ to be a Hölder map. Note also that our only assumptions on $r_{1}, r_{2}$ are that $r_{2}<1<r_{1}$ and that $\left\{z: r_{2}<|z|<r_{1}\right\}$ is conformally equivalent to $D$. Thus although $\beta(w)$ involves $r_{1}$, we are actually free to choose $r_{1}$ to be any number between 1 and $1 / r$, as long as we then take $r_{2}=r r_{1}$.

Next we use Theorem 5 to approximate $g^{-1}$ by $g_{1}^{-1}$. Recall the numbers $h_{1}, h_{2}$, $h=h_{1}+h_{2}$. Assume in addition that the right-hand arc $\gamma_{1}$ of $\partial G$ lies in the right 
half-plane and that the left-hand arc $\gamma_{2}$ lies in the left half-plane. To measure how "long" our quadrilateral $G$ is, we introduce the new quantities

$$
\sigma_{1}=\min _{w \in \gamma_{1}} \operatorname{Re} w, \quad \sigma_{2}=-\max _{w \in \gamma_{2}} \operatorname{Re} w, \quad \sigma=\sigma_{1}+\sigma_{2}, \quad \sigma_{*}=\min \left(\sigma_{1}, \sigma_{2}\right) .
$$

As we did before Corollary 2 , we now construct symmetric domains $D, D_{1}$ and maps $f, f_{1}$ with

$$
f(z)=e^{\pi g(1 / \pi) \log z)}, \quad f_{1}(z)=e^{\pi g_{1}((1 / \pi) \log z)}
$$

Clearly, then

$$
\rho_{1}=e^{\pi \sigma_{1}}, \quad \rho_{2}=e^{-\pi \sigma_{2}}, \quad \rho=e^{-\pi \sigma}, \quad \rho_{*}=e^{-\pi \sigma_{*}},
$$

and since $\rho \geq r=e^{-\pi h}$ we have that $h \geq \sigma$. Put

$$
K=\frac{1}{\pi} \log \kappa=\frac{1}{\pi} \log \frac{1-e^{-1.5 \pi} / 2}{4} \approx-0.44,
$$

and define

$$
b(w)=\frac{h_{1}-\operatorname{Re} g_{1}^{-1}(w)}{\sigma_{1}-K}, \quad w \in G .
$$

From Theorem 5 we immediately deduce that if $G$ is long, then $g_{1}^{-1}$ is a good approximation to $g^{-1}$ :

Corollary 6. If $\sigma_{*} \geq 1.5$, then

$$
\left|g^{-1}(w)-g_{1}^{-1}(w)\right| \leq \frac{1}{\pi}\left[25 e^{-\pi \sigma_{2}}\right]^{b(w)}\left[4.1 e^{-\pi \sigma}\right]^{1-b(w)} \leq \frac{25}{\pi} e^{-\pi \sigma_{2}}
$$

for all $w \in G$ with $\operatorname{Re} w>0$.

For example, if $\sigma_{1} \geq 1.5$ and $\sigma_{2} \geq 5$, then $g_{1}^{-1}(w)$ is within about $10^{-6}$ of $g^{-1}(w)$, provided $\operatorname{Re} w>0$.

In the special case that $\gamma_{2}$ is a vertical line segment, we obtain a much stronger result than Corollary 6 by reflecting $G$ in $\gamma_{2}$ to obtain a new quadrilateral that is "twice as long." In fact, by applying Corollary 6 to this new quadrilateral it is found that $\left|g^{-1}-g_{1}^{-1}\right| \leq(25 / \pi) e^{-\pi \sigma}$ in all of $G$.

Now assume that $h_{1}$ equals the conformal module of $Q_{1}$, so that we may define the map $q_{1}$, just as we did for Corollary 3. Define

$$
c(w)=\frac{h_{1}-\operatorname{Re} q_{1}^{-1}(w)}{\sigma_{1}-K}
$$

for $w \in G$ with $\operatorname{Re} w>0$. In Section 6 we prove the following estimates for approximating $g^{-1}$ by $q_{1}^{-1}$ : 
Corollary 7. If $\sigma_{*} \geq 1.5$, then

$$
\begin{gathered}
\left|g^{-1}(w)-q_{1}^{-1}(w)\right| \leq \frac{50}{\pi} e^{-\pi \sigma_{*}}, \\
\left|g^{-1}(w)-q_{1}^{-1}(w)\right| \leq \frac{2.8}{\pi}\left[25 e^{-\pi \sigma_{*}}\right]^{c(w)}\left[4.1 e^{-2 \pi \sigma_{*}}\right]^{1-c(w)}
\end{gathered}
$$

for all $w \in G$ with $\operatorname{Re} w>0$.

The order of approximation $O\left(e^{-\pi \sigma_{*}}\right)$ in Corollaries 6 and 7 is best possible, as we show by example in Section 7 .

\section{Questions for Future Consideration}

There seem to be two main ways to improve the results of this paper. The first is to find a better way than Theorem 4 of explicitly estimating both the Hölder class $\alpha$ of $f_{1}$ and its Hölder constant $M$ (particularly $M$ ) in terms of easily computable properties of $\gamma_{1}$. Once we have good estimates on $\alpha$ and $M$, of course, we may apply Corollaries 2 and 3.

The second way is to improve the $8 r$ Theorem (see below) that we use in proving Theorems 1 and 5. Instead of approximating $F$ in that theorem by just the identity map, the approximation in the outer half of the annulus could be improved by using the identity map multiplied by some "higher-order perturbation term."

\section{Proof of Theorem 1}

The main tool of the proof is the " $8 r$ Theorem" of Duren and Schiffer [DuS] and Gaier and Huckemann $[\mathrm{GaHu}]$ :

8r Theorem. If $F$ is a conformal map of $\{\zeta: r<|\zeta| \leq 1\}$ such that $0<|F(\zeta)| \leq 1$ for all $\zeta,|F(\zeta)|=1$ whenever $|\zeta|=1$, and $F(1)=1$, then

$$
\begin{aligned}
& \limsup _{\mid \zeta \zeta \rightarrow r}|F(\zeta)-\zeta|<5 r \\
& \sup _{r<|\zeta| \leq 1}|F(\zeta)-\zeta|<8 r .
\end{aligned}
$$

For alternative proofs of the theorem see $[\mathrm{GeH}],[\mathrm{Hu}]$, and $[\mathrm{Me}]$. We improve the $8 r$ Theorem somewhat:

5r Theorem. Suppose also that. $F$ is symmetric in the real axis. Then

$$
\begin{gathered}
\sup _{r<|\zeta| \leq 1}|F(\zeta)-\zeta|<5 r \\
\sup _{|\xi|=1}|F(\zeta)-\zeta|<4 r .
\end{gathered}
$$


To deduce the $5 r$ Theorem from the $8 r$ Theorem, it is enough to prove its second inequality. To prove this we start by taking $\zeta$ with $|\zeta|=1$ to maximize $|F(\zeta)-\zeta|$. Write $\zeta=e^{i \varphi}$ and $F(\zeta)=e^{i \theta}$. Since $F$ is symmetric in the real axis and $F(1)=1$, we may suppose $0 \leq \varphi, \theta \leq \pi$. Then $F$ maps the $\operatorname{arc}\left(e^{-i \varphi}, e^{i \varphi}\right)$ of the unit circle onto $\left(e^{-i \theta}, e^{i \theta}\right)$, and so

$$
|2 \theta-2 \varphi|<8 \arcsin r
$$

by Lemma 7 of $[\mathrm{GeH}]$. Hence

$$
|F(\zeta)-\zeta|=\left|e^{i \theta}-e^{i \varphi}\right|=2 \sin \frac{|\theta-\varphi|}{2} \leq 4 \sin \frac{|\theta-\varphi|}{4}<4 r
$$

which proves the $5 r$ Theorem.

Now we begin the proof of Theorem 1. Take $r_{0}=\min \left(e^{-\pi},\left(4^{\alpha} M\right)^{-1 / 2 \alpha}\right)$ and assume that $r_{*} \leq r_{0}$. We apply the $5 r$ Theorem to $F(\zeta)=\left(f_{1}^{-1} \circ f\right)\left(r_{1} \zeta\right) / r_{1}$ and deduce that

$$
\begin{aligned}
& \quad\left|\left(f_{1}^{-1} \circ f\right)(z)-z\right|<5 r_{2}, \quad r_{2}<|z|<r_{1}, \\
& \limsup _{|z| \rightarrow r_{1}}\left|\left(f_{1}^{-1} \circ f\right)(z)-z\right|<4 r_{2} .
\end{aligned}
$$

To prove (2.2) it is enough, by the Maximum Modulus Principle, to show that it holds for $|z|=1$ and $|z| \rightarrow r_{1}$. Suppose $|z|=1$. Then, by (3.1),

$$
\left|f(z)-f_{1}(z)\right|=\left|f_{1}\left(\left(f_{1}^{-1} \circ f\right)(z)\right)-f_{1}(z)\right| \leq \max _{|\zeta| \leq 1+5 r_{2}}\left|f_{1}^{\prime}(\xi)\right| 5 r_{2} .
$$

Put

$$
F_{1}(\zeta)=\frac{f_{1}\left(r_{1} \zeta\right)}{r_{1} f_{1}^{\prime}(0)}, \quad|\zeta|<1
$$

so that $F_{1}$ is holomorphic and univalent in the unit disk with $F_{1}(0)=0, F_{1}^{\prime}(0)=1$. Write $k(\zeta)=\zeta /(1-\zeta)^{2}$ for the Koebe function. Then the preceding inequality, the Distortion Theorem [Du, Theorem 2.5], and the fact that $r_{*} \leq r_{0} \leq e^{-\pi}$ combine to imply that

$$
\left|f(z)-f_{1}(z)\right| \leq \max _{|\zeta| \leq\left(1+5 r_{2}\right) / r_{1}}\left|F_{1}^{\prime}(\zeta)\right|\left|f_{1}^{\prime}(0)\right| 5 r_{2} \leq k^{\prime}\left(\left(1+5 e^{-\pi}\right) e^{-\pi}\right)\left|f_{1}^{\prime}(0)\right| 5 r_{2} .
$$

Hence

$$
\left|f(z)-f_{1}(z)\right| \leq(6.2)\left|f_{1}^{\prime}(0)\right| r_{2}, \quad|z|=1 .
$$

Next, the Growth Theorem [Du, Theorem 2.6] and the fact that $1 / r_{1} \leq e^{-\pi}$ imply that, for $|z|=1$,

$$
\left|f_{1}(z)\right|=r_{1}\left|F_{1}\left(\frac{z}{r_{1}}\right)\right|\left|f_{1}^{\prime}(0)\right| \geq r_{1}\left|k\left(\frac{-1}{r_{1}}\right)\right|\left|f_{1}^{\prime}(0)\right| \geq \frac{\left|f_{1}^{\prime}(0)\right|}{\left(1+e^{-\pi}\right)^{2}} .
$$


Hence

$$
\left|f_{1}(z)\right| \geq(0.9)\left|f_{1}^{\prime}(0)\right|, \quad|z|=1 \text {. }
$$

Since $r_{2} \leq e^{-\pi}$, we see from (3.3) and (3.4) that

$$
\left|f(z)-f_{1}(z)\right| \leq(0.3)\left|f_{1}(z)\right|, \quad|z|=1 .
$$

Observe now that, for each $z$,

$$
\log f(z)-\log f_{1}(z)=\int_{\left[f_{1}(z), f(z)\right]} \frac{1}{w} d w
$$

because the line segment $\left[f_{1}(z), f(z)\right]$ from $f_{1}(z)$ to $f(z)$ is contained in the domain of the logarithm. Thus for $|z|=1$ we conclude from (3.6), (3.5), (3.3), and (3.4) that

$$
\begin{aligned}
\left|\log f(z)-\log f_{1}(z)\right| & \leq \int_{\left[f_{1}(z), f(z)\right]} \frac{1}{|w|}|d w| \\
& \leq \frac{1}{(0.7)\left|f_{1}(z)\right|}\left|f(z)-f_{1}(z)\right| \\
& \leq \frac{(6.2)\left|f_{1}^{\prime}(0)\right| r_{2}}{(0.7)(0.9)\left|f_{1}^{\prime}(0)\right|} \\
& \leq 10 r_{2} .
\end{aligned}
$$

That is, (2.2) holds.

Next we deal with the case of $|z| \rightarrow r_{1}$. By (2.1) we have that

$$
\left|f(z)-f_{1}(z)\right|=\left|f_{1}\left(\left(f_{1}^{-1} \circ f\right)(z)\right)-f_{1}(z)\right| \leq M\left\|f_{1}\right\|_{\min } r_{1}^{-\alpha}\left|\left(f_{1}^{-1} \circ f\right)(z)-z\right|^{\alpha},
$$

and so from (3.2) we conclude that

$$
\underset{|z| \rightarrow r_{1}}{\lim \sup }\left|f(z)-f_{1}(z)\right| \leq 4^{\alpha} M r^{\alpha}\left\|f_{1}\right\|_{\min } .
$$

It follows that

$$
\limsup _{|z| \rightarrow r_{1}}\left|f(z)-f_{1}(z)\right| \leq\left\|f_{1}\right\|_{\min }
$$

since

$$
4^{\alpha} M r^{\alpha} \leq 4^{\alpha} M r_{*}^{2 \alpha} \leq 4^{\alpha} M\left(4^{\alpha} M\right)^{-1}=1 \text {. }
$$

In order to help us complete the proof, we proceed to show that

$$
\liminf _{|z| \rightarrow r_{1}} \inf _{w \in\left[f_{1}(z), f(z)\right]}|w| \geq \frac{\sqrt{3}}{2}\left\|f_{1}\right\|_{\min } .
$$


To show this, first note that by compactness we may suppose the "inf" and "lim inf" are achieved, i.e., we may reduce to showing that if

$$
|u| \geq\left\|f_{1}\right\|_{\min }, \quad|v| \geq\left\|f_{1}\right\|_{\min }, \quad|v-u| \leq\left\|f_{1}\right\|_{\text {min }},
$$

then

$$
\inf _{w \in[u, v]}|w| \geq \frac{\sqrt{3}}{2}\left\|f_{1}\right\|_{\min }
$$

here $u \approx f_{1}(z)$ and $v \approx f(z)$. Since $|v-u|^{2} \leq\left\|f_{1}\right\|_{\min }^{2}$, we certainly have that $\left\|f_{1}\right\|_{\min }^{2} \leq 2 u \cdot v$. Writing $w=t u+(1-t) v, 0 \leq t \leq 1$, we thus deduce that

$$
|w|^{2} \geq\left(1-t+t^{2}\right)\left\|f_{1}\right\|_{\min }^{2} \geq \frac{3}{4}\left\|f_{1}\right\|_{\min }^{2} \text {. }
$$

Obviously (3.9) follows.

Finally, by (3.6), (3.9), and (3.8),

$$
\begin{aligned}
\underset{|z| \rightarrow r_{1}}{\lim \sup \left|\log f(z)-\log f_{1}(z)\right|} & \leq \underset{|z| \rightarrow r_{1}}{\lim \sup _{\left.\mid f_{1}(z), f(z)\right]}} \frac{1}{|w|}|d w| \\
& \leq \frac{1}{\left\|f_{1}\right\|_{\min } \sqrt{3} / 2} \limsup _{|z| \rightarrow r_{1}}\left|f(z)-f_{1}(z)\right| \\
& \leq \frac{4^{\alpha} M r^{\alpha}\left\|f_{1}\right\|_{\text {min }}}{\left\|f_{1}\right\|_{\min } \sqrt{3} / 2} \\
& =\frac{2}{\sqrt{3}} 4^{\alpha} M r^{\alpha} .
\end{aligned}
$$

Now (2.2) follows from (3.7), (3.10), and the Maximum Modulus Principle. The proof of Theorem 1 is complete.

Proof of (2.4). Define

$H(z)=\log \left|\log f(z)-\log f_{1}(z)\right|-\left(1-\frac{\log |z|}{\log r_{1}}\right) \log \left(10 r_{2}\right)-\frac{\log |z|}{\log r_{1}} \log \left(\frac{2}{\sqrt{3}} 4^{\alpha} M r^{\alpha}\right)$

for $1 \leq|z|<r_{1}$, so that $H$ is subharmonic. Observe that $H(z) \leq 0$ when $|z|=1$ by (3.7) and that lim $\sup _{|z| \rightarrow r_{1}} H(z) \leq 0$ by (3.10). Hence $H(z) \leq 0$ by the Maximum Principle, and this implies (2.4).

Proof of Corollary 3 and (2.12). Take $h_{0}$ to be as in (2.11) and assume $h_{*} \geq h_{0}$. Extend $q_{1}$ by reflection in the imaginary axis to a conformal map of $\left(-h_{1}, h_{1}\right) \times$ $(0,1)$ onto $Q=\left\{x+i y:|x|+i y \in Q_{1}\right\} \cup(0, i)$. By applying $(2.8)$ with $G$ and $g$ replaced by $Q$ and $q_{1}$, respectively, we obtain that

$$
\left|q_{1}(z)-g_{1}(z)\right| \leq \frac{1}{\pi}\left[10 e^{-\pi h_{1}}\right]^{1-\operatorname{Re} z / h_{1}}\left[\frac{2}{\sqrt{3}} 4^{\alpha} M e^{-2 \pi h_{1} \alpha}\right]^{\operatorname{Re} z / h_{1}}, \quad z \in R, \quad \operatorname{Re} z>0 .
$$


Since $h_{i} \geq h_{*}$, this last inequality and (2.8) yield (2.12). Conclusion (2.10) of Corollary 3 is clearly a consequence of (2.12).

\section{Proof of Theorem 4}

Our proof involves the hyperbolic distance, so first we recall some of its properties. For $\Omega$ a proper, simply connected subdomain of the plane, we define the hyperbolic distance function on $\Omega$ to be

$$
h_{\Omega}\left(w_{1}, w_{2}\right)=\log \frac{1+\left|z_{1}-z_{2}\right| /\left|1-z_{1} \overline{z_{2}}\right|}{1-\left|z_{1}-z_{2}\right| /\left|1-z_{1} \overline{z_{2}}\right|},
$$

where $z_{i}=F^{-1}\left(w_{i}\right)$ and $F$ is any conformal map of the unit disk $\Delta$ onto $\Omega$. Later we use the Schwarz-Pick Lemma [Hi, Theorem 15.1.3], which states that holomorphic functions decrease hyperbolic distances: if $H: \Omega \rightarrow \Omega^{\prime}$ is holomorphic, then

$$
h_{\Omega^{\prime}}\left(H\left(w_{1}\right), H\left(w_{2}\right)\right) \leq h_{\Omega}\left(w_{1}, w_{2}\right), \quad w_{1}, w_{2} \in \Omega .
$$

Write $\delta\left(w_{1}\right)$ for the Euclidean distance from $w_{1} \in D_{1}$ to the boundary $\partial D_{1}$. The first step we take toward proving Theorem 4 is to establish that

$$
\alpha h_{D_{1}}\left(0, w_{1}\right)+\log \delta\left(w_{1}\right) \leq C+\pi \min x(y), \quad w_{1} \in D_{1},
$$

with

$$
C=\alpha \log 3+\pi \mathrm{Osc}\left(\gamma_{1}\right)+\max \left(0, \log \left(\pi \mathrm{Osc}\left(\gamma_{1}\right)+\log 2\right)\right) .
$$

To begin proving this, write $w_{1}=\left|w_{1}\right| e^{i \theta}$ with $-\pi<\theta \leq \pi$ and note that $\exp [\pi x(|\theta| / \pi)+i \theta]$ is the image of $x(|\theta| / \pi)+i(\theta / \pi)$ under the map $w \mapsto e^{\pi w}$, and thus is a boundary point of $D_{1}$. Let

$$
w_{2}=\frac{1}{2}\left\|f_{1}\right\|_{\min } e^{i \theta}=e^{-\log 2+\pi \min _{y} x(y)+i \theta} \in D_{1} .
$$

By taking $H(z)=z\left\|f_{1}\right\|_{\min },|z|<1$, we see from (4.2) that

$$
\max _{|w| \leq\left|w_{2}\right|} h_{D_{1}}(0, w) \leq \max _{|z| \leq 1 / 2} h_{\Delta}(0, z)=h_{\Delta}\left(0, \frac{1}{2}\right)=\log 3 .
$$

Thus (4.3) holds if $\left|w_{1}\right| \leq\left|w_{2}\right|$, because

$$
\begin{aligned}
\alpha h_{D_{1}}\left(0, w_{1}\right)+\log \delta\left(w_{1}\right) & \leq \alpha \log 3+\log \max _{w \in \partial D_{1}}|w| \\
& =\alpha \log 3+\pi \max _{y} x(y)=\alpha \log 3+\pi \operatorname{Osc}\left(\gamma_{1}\right)+\pi \min _{y} x(y) .
\end{aligned}
$$

Now suppose that $\left|w_{1}\right|>\left|w_{2}\right|$. Define a holomorphic function $H: \Delta \rightarrow D_{1}$ by

$$
H(z)=\exp \left[-\left[\pi x\left(\frac{|\theta|}{\pi}\right)-\pi \min _{y} x(y)+\log 2\right]\left(\frac{1-z}{1+z}\right)^{\alpha}+\pi x\left(\frac{|\theta|}{\pi}\right)+i \theta\right] ;
$$


to verify that $H$ maps $\Delta$ into $D_{1}$, observe first that the image of $H$ is exactly the image of the open cone

$$
\left\{\zeta:|\arg (\zeta)-\pi|<\frac{\pi \alpha}{2}\right\}+\left[x\left(\frac{|\theta|}{\pi}\right)+\frac{i \theta}{\pi}\right]
$$

under the map $\zeta \mapsto e^{\pi \zeta}$, and second that this cone does not intersect $\gamma_{1}$ since $\left|x^{\prime}(y)\right| \leq \cot (\pi \alpha / 2)$ for almost every $y$.

Clearly, $H(0)=w_{2}$. Also, if we define

$$
\eta=\frac{\pi x(|\theta| / \pi)-\log \left|w_{1}\right|}{\pi x(|\theta| / \pi)-\pi \min _{y} x(y)+\log 2},
$$

then $0<\eta<1$ since $\left|w_{2}\right|<\left|w_{1}\right|<e^{\pi x(|\theta| / \pi)}$. Defining $z_{1}$ by

$$
\left(\frac{1-z_{1}}{1+z_{1}}\right)^{\alpha}=\eta
$$

we have that $0<z_{1}<1$ and $H\left(z_{1}\right)=w_{1}$. Thus (4.2) and (4.1) imply that

$$
\begin{aligned}
& \alpha h_{D_{1}}\left(w_{2}, w_{1}\right)+\log \delta\left(w_{1}\right) \\
& \leq \alpha h_{\Delta}\left(0, z_{1}\right)+\log \delta\left(w_{1}\right) \\
&= \alpha \log \frac{1+z_{1}}{1-z_{1}}+\log \delta\left(w_{1}\right) \\
&= \log \frac{\pi x(|\theta| / \pi)-\pi \min _{y} x(y)+\log 2}{\pi x(|\theta| / \pi)-\log \left|w_{1}\right|}+\log \delta\left(w_{1}\right) \\
& \leq \log \left(\pi x\left(\frac{|\theta|}{\pi}\right)-\pi \min _{y} x(y)+\log 2\right)-\log \left(\log e^{\pi x(|\theta| / \pi)}-\log \left|w_{1}\right|\right) \\
&+\log \left(e^{\pi x(|\theta| / \pi)}-\left|w_{1}\right|\right) \\
& \leq \log \left(\pi x\left(\frac{|\theta|}{\pi}\right)-\pi \min _{y} x(y)+\log 2\right)+\log e^{\pi x(|\theta| / \pi)} \\
& \leq \log (\pi \max x(y)-\pi \min x(y)+\log 2)+\pi \max x(y) \\
& \leq \log \left(\pi \operatorname{Osc}\left(\gamma_{1}\right)+\log 2\right)+\pi \operatorname{Osc}\left(\gamma_{1}\right)+\pi \min _{y} x(y) .
\end{aligned}
$$

Estimate (4.3) follows, because

$$
h_{D_{1}}\left(0, w_{1}\right) \leq h_{D_{1}}\left(0, w_{2}\right)+h_{D_{1}}\left(w_{2}, w_{1}\right) \leq \log 3+h_{D_{1}}\left(w_{2}, w_{1}\right)
$$

by the triangle inequality.

Having established (4.3), we now essentially reproduce Becker and Pommerenke's proof of Theorem 1 [BeP] in order to get that $f_{1}$ is Hölder. 
Since $\delta\left(w_{1}\right) \geq r_{1}\left|f_{1}^{\prime}(z)\right|\left(1-\left|z / r_{1}\right|^{2}\right) / 4$ when $w_{1}=f_{1}(z)$ (see p. 22 of [Pol]), we have from (4.3) and (4.1) that

$$
\alpha \log \frac{1+\left|z / r_{1}\right|}{1-\left|z / r_{1}\right|}+\log \left\{r_{1}\left|f_{1}^{\prime}(z)\right| \frac{1-\left|z / r_{1}\right|^{2}}{4}\right\} \leq C+\pi \min _{y} x(y), \quad|z|<r_{1} .
$$

Hence

$$
r_{1}\left|f_{1}^{\prime}(z)\right| \leq \frac{4 e^{C} e^{\pi \min _{y} x(y)}}{\left(1-\left|z / r_{1}\right|\right)^{1-\alpha}}, \quad|z|<r_{1} .
$$

Put $F_{1}(\zeta)=f_{1}\left(r_{1} \zeta\right),|\zeta|<1$. Since $e^{\pi \min y x(y)}=\left\|f_{1}\right\|_{\min }$, we see that

$$
\left|F_{1}^{\prime}(\zeta)\right| \leq \frac{4 e^{C_{C}}\left\|f_{1}\right\|_{\min }}{\left(1-|\zeta|^{1-\alpha}\right.}, \quad|\zeta|<1 .
$$

Thus, by Lemma 8 below, $F_{1}$ is Hölder of order $\alpha$ with

$$
\left|F_{1}(\zeta)-F_{1}(\xi)\right| \leq M\left\|f_{1}\right\|_{\min }|\zeta-\xi|^{\alpha}, \quad|\zeta|,|\xi|<1,
$$

where

$$
M=4\left(1+\frac{2}{\alpha}\right) e^{c} .
$$

Since (2.14) follows easily, we have completed the proof of the theorem, except for proving the following lemma.

Lemma 8. Suppose $f$ is holomorphic in the unit disk, $f(0)=0$, and

$$
\left|f^{\prime}(z)\right| \leq \frac{K}{(1-|z|)^{1-\alpha}}, \quad|z|<1 .
$$

Then $f$ is Hölder of order $\alpha$, with

$$
|f(z)-f(w)| \leq\left(1+\frac{2}{\alpha}\right) K|z-w|^{\alpha}, \quad|z|,|w|<1 .
$$

That $f$ must be Hölder of order $\alpha$ was proved by Hardy and Littlewood in [HaL]; what we do here is to modify their proof somewhat so as to obtain the explicit Hölder constant in (4.4).

Proof of Lemma 8. First note that

$$
|f(z)| \leq \int_{[0, z]}\left|f^{\prime}(\zeta)\right||d \zeta| \leq \int_{0}^{1} \frac{K}{(1-t)^{1-\alpha}} d t=\frac{K}{\alpha} .
$$

Thus we may assume $|z-w|<1$ in proving (4.4). Write $z=|z| e^{i \theta}, w=|w| e^{i \varphi}$, and put

$$
s=1-|z-w|>0 .
$$

We may suppose $|w| \geq|z|$. 
If $s \geq|w|$, then

$$
|f(z)-f(w)| \leq \int_{[z, w]}\left|f^{\prime}(\zeta)\right||d \zeta| \leq \frac{K}{(1-s)^{1-\alpha}}|z-w|=K|z-w|^{\alpha},
$$

and so (4.4) holds. If $|z|<s<|w|$, then

$$
\begin{aligned}
|f(z)-f(w)| & \leq \int_{\left[z, s e^{i \varphi]}\right.}\left|f^{\prime}(\zeta)\right||d \zeta|+\int_{s}^{|w|}\left|f^{\prime}\left(t e^{i \varphi}\right)\right| d t \\
& \leq \frac{K}{(1-s)^{1-\alpha}}\left|z-s e^{i \varphi}\right|+\int_{s}^{1} \frac{K}{(1-t)^{1-\alpha}} d t \\
& \leq \frac{K}{(1-s)^{1-\alpha}}|z-w|+\frac{K}{\alpha}(1-s)^{\alpha} \\
& =\left(1+\frac{1}{\alpha}\right) K|z-w|^{\alpha},
\end{aligned}
$$

so that again (4.4) holds. Lastly, if $s \leq|z|$, then

$$
\begin{aligned}
|f(z)-f(w)| & \leq \int_{s}^{|z|}\left|f^{\prime}\left(t e^{i \theta}\right)\right| d t+\int_{\left[s e^{i \theta}, s e^{i \varphi}\right]}\left|f^{\prime}(\zeta)\right||d \zeta|+\int_{s}^{|w|}\left|f^{\prime}\left(t e^{i \varphi}\right)\right| d t \\
& \leq \frac{K}{\alpha}(1-s)^{\alpha}+\frac{K}{(1-s)^{1-\alpha}}\left|s e^{i \theta}-s e^{i \varphi}\right|+\frac{K}{\alpha}(1-s)^{\alpha} \\
& \leq\left(1+\frac{2}{\alpha}\right) K|z-w|^{\alpha},
\end{aligned}
$$

so that (4.4) holds once more. We have proved the lemma.

\section{An Example Showing Sharpness}

In this section we construct an example that shows the orders of approximation $O\left(r_{*}^{\min (1,2 \alpha)}\right)$ and $O\left(e^{-\pi h_{*} \min (1,2 \alpha)}\right)$ in Theorem 1 and Corollaries 2 and 3 are best possible, and that the Hölder exponent $\alpha$ in Theorem 4 is also best possible. At the end of the section we disprove two conjectures from [PaS1] and [PaS2].

Fix $\alpha \in(0,1]$. Define a function $H$ in the closed unit disk by

$$
H(\zeta)=\frac{i}{\alpha} \frac{1-((1+i \zeta) /(1-i \zeta))^{\alpha}}{1+((1+i \zeta) /(1-i \zeta))^{\alpha}}, \quad|\zeta| \leq 1 .
$$

When $\alpha=1, H$ is simply the identity map. To see that $H$ is also univalent when $\alpha<1$, and to understand its behavior, notice that

$$
\zeta \mapsto\left(\frac{1+i \zeta}{1-i \zeta}\right)^{\alpha}
$$




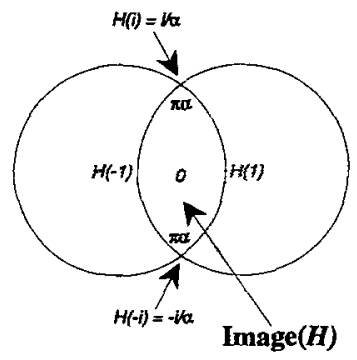

Fig. 6. The image of $H$ is the intersection of two disks.

maps the unit disk onto the sector $\{\xi:|\arg \xi|<\pi \alpha / 2\}$ in the right half-plane, and the map

$$
\xi \mapsto \frac{1-\xi}{1+\xi}
$$

takes that sector to the intersection of two disks, the boundary circles of which intersect at -1 and 1 , each making an angle $\pi \alpha / 2$ with the real axis at these points. Thus $H$ maps the unit disk to the domain pictured in Fig. 6 , and $H(0)=0$, $H(i)=i / \alpha$. Furthermore, $H$ is symmetric in the real and imaginary axes, and $H^{\prime}(0)=1, H^{\prime \prime}(0)=0$.

Take $0<r<1$ and put $r_{1}=1 / r^{1 / 2}, r_{2}=r^{1 / 2}, r_{*}=r^{1 / 2}$. Define

$$
D=\left\{H\left(\frac{\zeta+r / 2}{1+\zeta r / 2}\right): r<|\zeta|<1\right\}
$$

so that $D$ is a doubly connected domain containing neither the origin nor infinity and

$$
f(z)=H\left(\frac{z / r_{1}+r / 2}{1+\left(z / r_{1}\right) r / 2}\right)=H\left(\frac{r^{1 / 2} z+r / 2}{1+z r^{3 / 2} / 2}\right), \quad r_{2} \leq|z| \leq r_{1}
$$

maps $\left\{z: r_{2}<|z|<r_{1}\right\}$ conformally onto $D$. Now notice that

$$
D_{1}=D \cup K_{2}=\operatorname{Image}(H) .
$$

Thus

$$
f_{1}(z)=H\left(\frac{z}{r_{1}}\right), \quad|z| \leq r_{1},
$$

is the conformal map of $\left\{z:|z|<r_{1}\right\}$ onto $D_{1}$ with $f_{1}(0)=0$ and $\left(f_{1}^{-1} \circ f\right)\left(r_{1}\right)=r_{1}$. Note that both $f$ and $f_{1}$ are symmetric in the real axis and are positive on the positive real axis.

In order for Theorem 1 to apply, we want $f_{1}$ to be Hölder of order $\alpha$. What is 
more, for the purposes of this example we need to show that the Hölder constant $M$ in (2.1) is independent of $r$. To establish all this, first calculate

$$
H^{\prime}(\zeta)=\frac{4}{(1-i \zeta)^{1+\alpha}\left[1+((1+i \zeta) /(1-i \zeta))^{\alpha}\right]^{2}(1+i \zeta)^{1-\alpha}} .
$$

Clearly,

$$
\left|H^{\prime}(\zeta)\right| \leq \frac{4}{(1-|\zeta|)^{1-\alpha}}, \quad|\zeta|<1, \quad \operatorname{Im} \zeta \geq 0
$$

and the same inequality holds by symmetry when $\operatorname{Im} \zeta \leq 0$. Lemma 8 in Section 4 now gives that $H$ is Hölder of order $\alpha$ with a Hölder constant depending only on $\alpha$. Since $\left\|f_{1}\right\|_{\min } \geq \frac{1}{4}$ by the Koebe $\frac{1}{4}$-Theorem applied to $H$, we deduce that (2.1) holds with a constant $M$ depending only on $\alpha$, as desired.

Next we show that the order of approximation $O\left(r_{*}^{\min (1,2 \alpha)}\right)$ in (2.2) of Theorem 1 is best possible. For this, notice first that

$$
\begin{aligned}
\underset{1 \leq|z| \leq r_{1}}{\max \left|\log f(z)-\log f_{1}(z)\right|} & \geq\left|\operatorname{Im} \log f\left(i r_{1}\right)-\operatorname{Im} \log f_{1}\left(i r_{1}\right)\right| \\
& =\left|\operatorname{Im} \log H\left(\frac{i+r / 2}{1+i r / 2}\right)-\operatorname{Im} \log H(i)\right| \\
& =\left|\operatorname{Im} \log \frac{i}{\alpha} \frac{1-(i r / 2)^{\alpha}}{1+(i r / 2)^{\alpha}}-\operatorname{Im} \log \frac{i}{\alpha}\right| \\
& \geq M^{\prime \prime} r^{\alpha}=M^{\prime \prime} r_{*}^{2 \alpha}
\end{aligned}
$$

for all small $r$, where $M^{\prime \prime}>0$ depends only on $\alpha$. (It is for our later use that we have considered "Im $\log$ " here instead of just "log.") Second, because $H(\zeta)=$ $\zeta+O\left(\zeta^{3}\right)$ near the origin,

$$
\begin{aligned}
\max _{1 \leq|z| \leq r_{1}}\left|\log f(z)-\log f_{1}(z)\right| & \geq\left|\log f(1)-\log f_{1}(1)\right| \\
& =\left|\log H\left(\frac{r^{1 / 2}+r / 2}{1+r^{3 / 2} / 2}\right)-\log H\left(r^{1 / 2}\right)\right| \\
& =\left|\log \left(r^{1 / 2}+\frac{r}{2}+O\left(r^{2}\right)\right)-\log \left(r^{1 / 2}+O\left(r^{3 / 2}\right)\right)\right| \\
& \geq M^{\prime \prime} r^{1 / 2}=M^{\prime \prime} r_{*}
\end{aligned}
$$

for all small $r$, for some $M^{\prime \prime}>0$. The previous two inequalities taken together prove by example that the order of approximation $O\left(r_{*}^{\min (1,2 \alpha)}\right)$ in Theorem 1 is best possible, as $r_{*} \rightarrow 0$.

The very same example shows that the order of approximation $O\left(e^{-\pi h_{*} \min (1,2 \alpha)}\right)$ 
in Corollary 2 is also best possible. That is, we take

$$
\begin{aligned}
h & =\left(\frac{1}{\pi}\right) \log \left(\frac{1}{r}\right), \quad h_{1}=\frac{h}{2}=h_{2}, \quad h_{*}=\frac{h}{2}, \\
R & =\left(-h_{2}, h_{1}\right) \times(0,1), \quad G=\left\{\left(\frac{1}{\pi}\right) \log w: w \in D, \operatorname{Im} w>0\right\}, \\
g(z) & =\left(\frac{1}{\pi}\right) \log f\left(e^{\pi z}\right), \quad z \in R, \\
R_{1} & =\left(-\infty, h_{1}\right) \times(0,1), \quad G_{1}=\left\{\left(\frac{1}{\pi}\right) \log w: w \in D_{1}, \operatorname{Im} w>0\right\}, \\
g_{1}(z) & =\left(\frac{1}{\pi}\right) \log f_{1}\left(e^{\pi z}\right), \quad z \in R_{1} .
\end{aligned}
$$

Given all of this, we have from above that

$$
\begin{aligned}
\left|\operatorname{Im} g\left(h_{1}+\frac{i}{2}\right)-\operatorname{Im} g_{1}\left(h_{1}+\frac{i}{2}\right)\right| & \geq \frac{M^{\prime \prime}}{\pi} e^{-2 \pi h_{*} \alpha}, \\
\left|g(0)-g_{1}(0)\right| & \geq \frac{M^{\prime \prime}}{\pi} e^{-\pi h_{*}}
\end{aligned}
$$

provided $h_{*}$ is large, i.e., provided $r$ is small. Here $M^{\prime \prime}$ depends only on $\alpha$. Hence the order of approximation in Corollary 2 is best possible, as $h_{*} \rightarrow \infty$.

Corollary 3 is sharp too, but a few extra steps are needed to see this. First, remember that we need $\gamma_{2}$ and $\gamma_{1}$ to lie in the left and right half-planes, respectively. As long as $r$ is small, this can be achieved by replacing $G, g, G_{1}, g_{1}$ with their translates $G+\lambda, g+\lambda, G_{1}+\lambda, g_{1}+\lambda$ for some real constant $\lambda$. Note that this does not change $h_{1}, h_{2}, R$, or $R_{1}$, and that although $f_{1}$ must be replaced by $e^{\pi \lambda} f_{1}$, this map has the same Hölder constant $M$ as $f_{1}$.

Next, in view of the continuous dependence of the module of $Q_{1}$ on $\partial Q_{1}$ (see p. 26 of [LeV]), we may choose $\lambda$ so that the module of $Q_{1}$ equals $h_{1}$, as required for Corollary 3. Notice that $\lambda \rightarrow \infty$ as $h_{1} \rightarrow \infty$.

Now we remark that by the symmetry of $\partial D_{1}$ in the imaginary axis, $\gamma_{1}$ is symmetric in the line $\left\{w: \operatorname{Im} w=\frac{1}{2}\right\}$, so that both $q_{1}$ and $g_{1}$ are also symmetric in that line. Hence

$$
\left.q_{1}\left(h_{1}+\frac{i}{2}\right)=\text { (midpoint of } \gamma_{1}\right)=g_{1}\left(h_{1}+\frac{i}{2}\right)
$$


and so

$$
\begin{aligned}
\left|\operatorname{Im} g\left(h_{1}+\frac{i}{2}\right)-\operatorname{Im} q_{1}\left(h_{1}+\frac{i}{2}\right)\right| & =\left|\operatorname{Im} g\left(h_{1}+\frac{i}{2}\right)-\operatorname{Im} g_{1}\left(h_{1}+\frac{i}{2}\right)\right| \\
& \geq \frac{M^{\prime \prime}}{\pi} e^{-2 \pi h_{*} \alpha}
\end{aligned}
$$

for $r$ sufficiently small, by (5.1).

Furthermore, $q_{1}(0)=0$ by definition of $q_{1}$ and so

$$
\left|g(0)-q_{1}(0)\right|=\left|\left(\frac{1}{\pi}\right) \log f\left(e^{\pi 0}\right)+\lambda-0\right|=\left|\left(\frac{1}{\pi}\right) \log \left[r^{1 / 2}+\frac{r}{2}+O\left(r^{2}\right)\right]+\lambda\right| .
$$

However,

$$
\left(\frac{1}{\pi}\right) \log \frac{1}{r^{1 / 2}}=h_{1}=\bmod \left(Q_{1}\right)
$$

while by Theorem 4 of [GaH2]

$$
\bmod \left(Q_{1}\right)=\left[\lambda+\left(\frac{1}{\pi}\right) \log H(1)\right]+\left(\frac{1}{\pi}\right) \log R+O\left(e^{-2 \pi[\lambda+(1 / \pi) \log H(1)]}\right)
$$

for some fixed number $R$ depending only on $\alpha$. It is found that $R=1 / H(1)$; thus

$$
\left(\frac{1}{\pi}\right) \log \frac{1}{r^{1 / 2}}=\bmod \left(Q_{1}\right)=\lambda+O\left(e^{-2 \pi \lambda}\right)
$$

In particular, this implies that $-2 \pi \lambda \leq \log r+1$ for all large $\lambda$. Hence we have from the last equation that

$$
\lambda=\left(\frac{1}{\pi}\right) \log \frac{1}{r^{1 / 2}}-O(r)
$$

We conclude that

$$
\begin{aligned}
\left|g(0)-q_{1}(0)\right| & =\left|\left(\frac{1}{\pi}\right) \log \left[r^{1 / 2}+\frac{r}{2}+O\left(r^{2}\right)\right]+\left(\frac{1}{\pi}\right) \log \frac{1}{r^{1 / 2}}-O(r)\right| \\
& \geq M^{\prime \prime} r^{1 / 2}=M^{\prime \prime} e^{-\pi h_{*} .}
\end{aligned}
$$

Combining this estimate with (5.2), we obtain that the order of approximation in Corollary 3 is best possible, as $h_{*} \rightarrow \infty$.

To establish the sharpness of the Hölder exponent $\alpha$ in Theorem 4 we first verify that in the example constructed above for Corollary $2, \gamma_{1}$ does indeed satisfy the hypothesis (2.13); later we show our $f_{1}$ is not Hölder for any order greater than $\alpha$.

We have from our construction that

$$
\gamma_{1}=\left\{\left(\frac{1}{\pi}\right) \log w: w \in \partial D_{1}, \operatorname{Im} w \geq 0\right\},
$$


where $\partial D_{1}$ is made up of two circular arcs, as in Figure 6. Parametrizing the right-hand arc $\Gamma$ as $\rho(\theta) e^{i \theta},-\pi / 2 \leq \theta \leq \pi / 2$, we see that $x(y)=(1 / \pi) \log \rho(\pi y)$ for $0 \leq y \leq \frac{1}{2}$. By symmetry, then, (2.13) would be implied by

$$
0 \leq(\log \rho)^{\prime}(\theta) \leq \cot \left(\frac{\pi \alpha}{2}\right), \quad 0<\theta<\frac{\pi}{2} .
$$

The left-hand inequality is geometrically obvious. To prove the right-hand inequality, observe that

$$
\arg \left(\frac{d}{d \theta} \rho(\theta) e^{i \theta}\right)-\theta=\cot ^{-1}\left(\frac{\rho^{\prime}(\theta)}{\rho(\theta)}\right) .
$$

The left-hand side of this equation gives the angle between the tangent and radius vectors at $\theta$, and this is easily seen to be minimal when $\theta=\pi / 2$. Thus $\rho^{\prime}(\theta) / \rho(\theta)$ is maximal for $\theta=\pi / 2$. Hence

$$
\begin{aligned}
(\log \rho)^{\prime}(\theta) & =\frac{\rho^{\prime}(\theta)}{\rho(\theta)} \\
& \leq \frac{\rho^{\prime}(\pi / 2)}{\rho(\pi / 2)}=\cot \left[\left(\frac{\pi}{2}+\frac{\pi \alpha}{2}\right)-\frac{\pi}{2}\right]=\cot \left(\frac{\pi \alpha}{2}\right),
\end{aligned}
$$

which proves (5.3). It follows that $\gamma_{1}$ satisfies (2.13), as desired.

We still want to show that $f_{1}$ is Hölder of no order higher than $\alpha$. This is easy, however, since by direct computation

$$
\left|f_{1}\left(i r_{1}\right)-f_{1}\left(t i r_{1}\right)\right|=|H(i)-H(i t)| \approx \frac{2^{1-\alpha}}{\alpha}(1-t)^{\alpha}=\frac{2^{1-\alpha}}{\alpha r_{1}^{\alpha}}\left|i r_{1}-t i r_{1}\right|^{\alpha}
$$

as $t \rightarrow 1-$.

Our final task for this section is to disprove the conjectures of Papamichael and Stylianopoulos [PaS1, Conjectures 3.1 and 3.2], [PaS2, Remarks 5.2 and 5.4] that the two estimates

$$
\begin{gathered}
\max _{z \in \bar{R}, \operatorname{Re} z \geq 0}\left|g(z)-q_{1}(z)\right|=O\left(e^{-\pi h_{*}}\right), \\
\max _{y}\left|\operatorname{Im} g\left(h_{1}+i y\right)-\operatorname{Im} q_{1}\left(h_{1}+i y\right)\right|=O\left(e^{-2 \pi h_{*}}\right)
\end{gathered}
$$

hold as $h_{*} \rightarrow \infty$ whenever $\gamma_{1}$ and $\gamma_{2}$ can be parametrized as

$$
\gamma_{1}=x(y)+i y, \quad \gamma_{2}(y)=x_{2}(y)+i y,
$$

with $x, x_{2}$ absolutely continuous and

$$
\text { ess } \sup _{y}\left|x^{\prime}(y)\right|<\infty, \quad \text { ess } \sup _{y}\left|x_{2}^{\prime}(y)\right|<\infty .
$$


To see that (5.4) and (5.5) are false, simply take $0<\alpha<\frac{1}{2}$ and recall (5.2). Our only remaining task is to verify the hypothesis that $\gamma_{2}$ can be parametrized as $x_{2}(y)+i y$ with $x_{2}$ having bounded derivative; we have previously done this for $\gamma_{1}$. Recall that $\gamma_{2}=(1 / \pi) \log \Gamma_{2}+\lambda$, where

$$
\Gamma_{2}=\left\{H\left(\frac{r e^{i \varphi}+r / 2}{1+r^{2} e^{i \varphi} / 2}\right): 0 \leq \varphi \leq \pi\right\}
$$

is the upper half of the inner boundary of $D$. What we wish to show is that $\Gamma_{2}$ may be parametrized as $\rho_{2}(\theta) e^{i \theta}$ with $\log \rho_{2}$ having bounded derivative. Toward this goal, write

$$
\theta(\varphi)=\arg H\left(\frac{r e^{i \varphi}+r / 2}{1+r^{2} e^{i \varphi} / 2}\right)=\operatorname{Im} \log H\left(\frac{r e^{i \varphi}+r / 2}{1+r^{2} e^{i \varphi} / 2}\right)
$$

and deduce (since $\left.H^{\prime}(0)=1\right)$ that

$$
\frac{d \theta}{d \varphi}=\operatorname{Im} i \frac{1+O(r)}{1+e^{-i \varphi} / 2+O(r)}>0
$$

for all $\varphi$, provided $r$ is small enough. Thus we can invert $\theta(\varphi)$ to obtain $\varphi=\varphi(\theta)$, and so

$$
\Gamma_{2}=\left\{\rho_{2}(\theta) e^{i \theta}: 0 \leq \theta \leq \pi\right\}
$$

with

$$
\rho_{2}(\theta)=\left|H\left(\frac{r e^{i \varphi(\theta)}+r / 2}{1+r^{2} e^{i \varphi(\theta)} / 2}\right)\right| .
$$

Clearly, $\log \rho_{2}$ has bounded derivative, and this completes the disproof of conjectures (5.4) and (5.5).

Actually, one final comment is pertinent. For their conjectures, Papamichael and Stylianopoulos used the definition

$$
h_{*}=\min \left(\bmod \left(Q_{1}\right), \bmod \left(Q_{2}\right)\right)
$$

rather than our definition $h_{*}=\min \left(h_{1}, h_{2}\right)$. No harm is done, though, because the two definitions are equivalent for the purposes of (5.4) and (5.5): we took $h_{1}=\bmod \left(Q_{1}\right)$, and

$$
h_{2}=h-h_{1}=\bmod (G)-\bmod \left(Q_{1}\right)=\bmod \left(Q_{2}\right)+O(1)
$$

as $r \rightarrow 0$, by Theorem 2 of [GaH2].

\section{Proof of Theorem 5}

Writing $w=f_{1}(z)$, we can restate conclusion (2.15) of the theorem as

$$
\left|\log \left(f^{-1} \circ f_{1}\right)(z)-\log z\right| \leq\left[25 \rho_{2}\right]^{\beta(z)}[4.1 \rho]^{1-\beta(z)},
$$


where $|z|<r_{1},\left|f_{1}(z)\right|>1$, and " $\beta(z)$ " means $\beta\left(f_{1}(z)\right)$. The condition $\left|f_{1}(z)\right|>1$ prevents $|z|$ from being too small; to make this precise, put

$$
F_{1}(\zeta)=\frac{f_{1}\left(r_{1} \zeta\right)}{r_{1} f_{1}^{\prime}(0)}, \quad|\zeta|<1,
$$

so that $F_{1}$ is holomorphic and univalent in the unit disk with $F_{1}(0)=0, F_{1}^{\prime}(0)=1$. Write $k(\zeta)=\zeta /(1-\zeta)^{2}$ for the Koebe function. Take $|z|<r_{1}$ with $\left|f_{1}(z)\right| \geq 1$. Then, by the Growth Theorem [Du, Theorem 2.6],

$$
|z|=\left|f_{1}^{-1}\left(f_{1}(z)\right)\right|=r_{1}\left|F_{1}^{-1}\left(\frac{f_{1}(z)}{r_{1} f_{1}^{\prime}(0)}\right)\right| \geq r_{1} k^{-1}\left(\frac{\left|f_{1}(z)\right|}{r_{1}\left|f_{1}^{\prime}(0)\right|}\right) \geq r_{1} k^{-1}\left(\frac{1}{r_{1}\left|f_{1}^{\prime}(0)\right|}\right) .
$$

Also, by the Koebe $\frac{1}{4}$-Theorem [Du, Theorem 2.3] applied to $F_{1}$ we have that

$$
\frac{1}{r_{1}\left|f_{1}^{\prime}(0)\right|} \geq \frac{1}{4\left\|f_{1}\right\|_{\min }}=\frac{1}{4 \rho_{1}}
$$

and so

$$
|z| \geq r_{1} k^{-1} \frac{1}{4 \rho_{1}} .
$$

In general it is true that $k^{-1}(t) \geq t(1-2 t)$, and thus

$$
\frac{|z|}{r_{1}} \geq \frac{1-2 / 4 \rho_{1}}{4 \rho_{1}} \geq \frac{1-1 / 2 e^{1.5 \pi}}{4 \rho_{1}}=\frac{\kappa}{\rho_{1}} .
$$

We have just shown that if $\left|f_{1}(z)\right| \geq 1$, then $|z| / r_{1} \geq \kappa / \rho_{1}$; that is,

$$
\beta(z) \leq 1 .
$$

We need a partial converse to this, so now suppose that $\kappa / \rho_{1} \leq|z| / r_{1}<1$. We show that

$$
\left|f_{1}(z)\right|>\rho_{2}=\underset{w \in K_{z}}{\max }|w|,
$$

from which it follows in particular that $f_{1}(z) \in D$.

In order to prove (6.2), observe that, by the Growth Theorem,

$$
\left.\left|f_{1}(z)\right|=r_{1}\left|f_{1}^{\prime}(0)\right|\left|F_{1}\left(\frac{z}{r_{1}}\right)\right| \geq r_{1}\left|f_{1}^{\prime}(0)\right|\left|k\left(\frac{-|z|}{r_{1}}\right)\right| \geq r_{1}\left|f_{1}^{\prime}(0)\right| \mid k\left(\frac{-\kappa}{\rho_{1}}\right)\right\} \text {. }
$$

Now, $r_{1}\left|f_{1}^{\prime}(0)\right| \geq \rho_{1}$ by Schwarz's Lemma applied to the function

$$
\xi \mapsto \frac{f_{1}^{-1}\left(\rho_{1} \xi\right)}{r_{1}},
$$


and so

$$
\left|f_{1}(z)\right| \geq \rho_{1}\left|k\left(\frac{-\kappa}{\rho_{1}}\right)\right|=\frac{\kappa}{\left(1+\kappa / \rho_{1}\right)^{2}} \geq \frac{\kappa}{\left(1+\kappa / e^{1.5 \pi}\right)^{2}}>0.24>\rho_{2},
$$

which is (6.2).

What we have shown so far is that it suffices to prove $(6.1)$ for $z$ with $\kappa / \rho_{1} \leq|z| / r_{1}<1$, and that the function $f^{-1} \circ f_{1}$ is indeed defined for all such $z$, by $(6.2)$.

Now recall the $5 r$ Theorem in Section 3, which yielded (3.1) and (3.2). From those two results we obtain that

$$
\left|\left(f^{-1} \circ f_{1}\right)(z)-z\right|<5 r_{2}, \quad \frac{\kappa}{\rho_{1}} \leq \frac{|z|}{r_{1}}<1
$$

$$
\lim \sup \left|\left(f^{-1} \circ f_{1}\right)(z)-z\right|<4 r_{2} \text {. }
$$

$$
|z| \rightarrow r_{1}
$$

Using (6.3) and the fact that $\rho \geq r, \rho_{2} \leq e^{-1.5 \pi}$, we see that, for $\kappa / \rho_{1} \leq|z| / r_{1}<1$,

$$
\begin{aligned}
\left|\log \left(f^{-1} \circ f_{1}\right)(z)-\log z\right| & \leq \int_{\left[z,\left(f^{-1} \circ f_{1}\right)(z)\right]} \frac{1}{|\xi|}|d \xi| \\
& \leq \frac{1}{r_{1} \kappa / \rho_{1}-5 r_{2}}\left|\left(f^{-1} \circ f_{1}\right)(z)-z\right| \\
& \leq \frac{1}{\left(\kappa \rho / r-5 \rho_{2}\right)\left(r_{2} / \rho_{2}\right)} 5 r_{2} \\
& \leq \frac{5 \rho_{2}}{\kappa-5 \rho_{2}} \\
& <25 \rho_{2} .
\end{aligned}
$$

Also, from (6.4) we deduce that

(6.6) $\limsup _{|z| \rightarrow r_{1}}\left|\log \left(f^{-1} \circ f_{1}\right)(z)-\log z\right| \leq \limsup _{|z| \rightarrow r_{1}} \int_{\left[z_{1}\left(f^{\left.\left.-1 \circ f_{1}\right)(z)\right]}\right.\right.} \frac{1}{|\xi|}|d \xi|$

$$
\begin{aligned}
& \leq \frac{1}{r_{1}-4 r_{2}} \limsup _{|z| \rightarrow r_{1}}\left|\left(f^{-1} \circ f_{1}\right)(z)-z\right| \\
& \leq \frac{4}{1-4 r} r \\
& \leq 4.1 r \leq 4.1 \rho .
\end{aligned}
$$


Finally, define

$$
\begin{aligned}
H(z)= & \log \left|\log \left(f^{-1} \circ f_{1}\right)(z)-\log z\right|-\frac{\log \left(r_{1} /|z|\right)}{\log \left(\rho_{1} / \kappa\right)} \log \left[25 \rho_{2}\right] \\
& -\left(1-\frac{\log \left(r_{1} /|z|\right)}{\log \left(\rho_{1} / \kappa\right)}\right) \log [4.1 \rho]
\end{aligned}
$$

so that $H$ is subharmonic for $\kappa / \rho_{1} \leq|z| / r_{1}<1$. Clearly, (6.5) implies that $H(z) \leq 0$ when $|z| / r_{1}=\kappa / \rho_{1}$, and (6.6) implies that lim $\sup _{|z| \rightarrow r_{1}} H(z) \leq 0$. Hence $H(z) \leq 0$ for all $z$ by the Maximum Principle. Inequality (6.1) follows, and the theorem is proved.

Proof of Corollary 7. Extend $q_{1}$ by reflection in the imaginary axis to a conformal map of $\left(-h_{1}, h_{1}\right) \times(0,1)$ onto $Q=\left\{x+i y:|x|+i y \in Q_{1}\right\} \cup(0, i)$. By applying (2.16) with $G$ and $g$ replaced by $Q$ and $q_{1}$, respectively, we obtain that

$$
\left|q_{1}^{-1}(w)-g_{1}^{-1}(w)\right| \leq \frac{1}{\pi}\left[25 e^{-\pi \sigma_{1}}\right]^{b(w)}\left[4.1 e^{-2 \pi \sigma_{1}}\right]^{1-b(w)} \leq \frac{25}{\pi} e^{-\pi \sigma_{1}}
$$

for $w \in G, \operatorname{Re} w>0$. Since $\sigma_{i} \geq \sigma_{*}$, this last inequality and (2.16) give that

$$
\left|g^{-1}(w)-q_{1}^{-1}(w)\right| \leq \frac{2}{\pi}\left[25 e^{-\pi \sigma_{*}}\right]^{b(w)}\left[4.1 e^{-2 \pi \sigma_{*}}\right]^{1-b(w)} \leq \frac{2}{\pi} 25 e^{-\pi \sigma_{*}}=\frac{50}{\pi} e^{-\pi \sigma_{*}}
$$

for $w \in G, \operatorname{Re} w>0$, which gives (2.17) and also (2.18) except that we have " $b(w)$ " rather than " $c(w)$ " on the right-hand side. To derive (2.18) from the preceding inequality, just observe that, for $w \in G$ with $\operatorname{Re} w>0$,

$$
\begin{aligned}
& {\left[25 e^{-\pi \sigma_{*}}\right]^{[b(w)-c(w)]}\left[4.1 e^{-2 \pi \sigma_{*}}\right]^{-[b(w)-c(w)]}=\left(\frac{25}{4.1} e^{\pi \sigma_{*}}\right)^{\operatorname{Re}\left(q^{-1}(w)-g_{1}^{-1}(w)\right) /\left(\sigma_{1}-K\right)}} \\
& \leq\left(7 e^{\pi \sigma_{*}}\right)^{\left(25 e^{\left.-\pi \sigma_{1} / 1.5 \pi\right)}\right.} \\
& \leq 7^{\left(25 e^{-1.5 \pi / 1.5 \pi)}\right.} \exp \left[\frac{25}{1.5} \sigma_{*} e^{-\pi \sigma_{*}}\right] \\
& \leq(1.1) \exp \left[25 e^{-1.5 \pi}\right]<1.4 \text {. }
\end{aligned}
$$

Since $(2 / \pi)(1.4)=2.8 / \pi$, the corollary is proved.

\section{Another Example Showing Sharpness}

In this final section we construct an example that shows the orders of approximation $O\left(\rho_{*}\right)$ and $O\left(e^{-\pi \sigma_{*}}\right)$ in Theorem 5 and Corollaries 6 and 7 are best possible. 
Take $r>0$ to be small and put $r_{1}=1 / r^{1 / 2}, r_{2}=r^{1 / 2}, r_{*}=r^{1 / 2}$. Define

$$
D=\left\{r_{1} \frac{\zeta+r / 2}{1+\zeta r / 2}: r<|\zeta|<1\right\},
$$

so that $D$ contains neither the origin nor infinity and

$$
f(z)=r_{1} \frac{z / r_{1}+r / 2}{1+\left(z / r_{1}\right) r / 2}=\frac{z+r^{1 / 2} / 2}{1+z r^{3 / 2} / 2}
$$

is a conformal map of $\left\{z: r_{2}<|z|<r_{1}\right\}$ onto $D$. Both $D$ and $f$ are symmetric in the real axis and $f$ is positive on the positive real axis. Also, a conformal map (namely, the identity) of $\left\{z: 1<|z|<r_{1}\right\}$ onto $D \cap\{w:|w|>1\}$ exists. Note that $\rho_{2}<2 r^{1 / 2}$

Since

$$
D_{1}=D \cup K_{2}=\left\{w:|w|<r_{1}\right\}
$$

and $f\left(r_{1}\right)=r_{1}$, the map $f_{1}$ is simply the identity:

$$
f_{1}(z)=z, \quad|z|<r_{1} .
$$

The inverse maps are

$$
f^{-1}(w)=\frac{w-r^{1 / 2} / 2}{1-w r^{3 / 2} / 2}, \quad f_{1}^{-1}(w)=w .
$$

Hence

$$
\begin{aligned}
\sup _{w \in D,|w|>1}\left|\log f^{-1}(w)-\log f_{1}^{-1}(w)\right| & \geq\left|\log f^{-1}(1)-\log f_{1}^{-1}(1)\right| \\
& =\left|\log \frac{1-r^{1 / 2} / 2}{1-r^{3 / 2} / 2}-\log 1\right| \\
& \geq M^{\prime \prime} r^{1 / 2}>\frac{M^{\prime \prime}}{2} \rho_{2}
\end{aligned}
$$

for all small $r$, where $M^{\prime \prime}>0$. Letting $r \rightarrow \tilde{0}$, we see that the order of approximation $O\left(\rho_{2}\right)$ in Theorem 5 is best possible.

By "lifting" this example with the map $w \mapsto(1 / \pi) \log w$, we deduce that the orders of approximation $O\left(e^{-\pi \sigma_{2}}\right), O\left(e^{-\pi \sigma_{*}}\right)$ in Corollaries 6 and 7 are also best possible. Note that $g_{1}$ is the lift of the identity map $f_{1}$ and thus is also the identity, and that $q_{1}$ is the identity since $Q_{1}$ is a rectangle. We leave the details to the reader.

Acknowledgments. This research was begun while partially supported by a Grant-in-Aid of Research from Sigma Xi, the Scientific Research Society. I would like to thank Dr. N. Papamichael for suggesting these problems to me, and Dr. James Jenkins for making several helpful comments along the way. Thanks are due also to my advisor Albert Baernstein II, who has provided invaluable guidance 
during the past 5 years. This research was sparked by a conversation with Walter Hayman that I enjoyed while visiting the University of York, England. I am grateful to the referee for making a number of detailed suggestions; these have significantly improved the paper.

\section{References}

[BeP] J. BECKER, CH. POMMERENKE (1982): Hölder continuity of conformal maps and non-quasiconformal Jordan curves. Comment. Math. Helv., 57:221-225.

[DeLE] T. K. DELILlo, A. R. ElCRAT (1993): A Fornberg-like conformal mapping method for slender regions J. Comput. Appl. Math., 46:49-64.

[Du] P. L. Duren (1983): Univalent Functions. New York: Springer-Verlag.

[DuS] P. L. DUREN, M. SCHIFFER (1962): A variational method for functions schlicht in an annulus. Arch. Rational Mech. Anal., 9:260-272.

[Ga] D. GAIER (1972): Estimates of conformal mappings near the boundary. Indiana Math. J., 21:581-595.

[GaH1] D. GAIER, W. K. HAYMAN (1990): Moduli of long quadrilaterals and thick ring domains. Rend. Mat. Appl. (7), 10:809-834.

[GaH2] D. GAIER, W. K. HAYMAN (1991): On the computation of modules of long quadrilaterals. Constr. Approx., 7:453-467.

[GaHu] D. GAIER, F. HuCKEMANN (1962): Extremal problems for functions schlicht in an annulus. Arch. Rational Mech. Anal., 9:415-421.

[GaP] D. Galer, N. PAPAMIChael (1987): On the comparison of two numerical methods for conformal mapping. IMA J. Numer. Anal., 7:261-282.

[GeH] F. W. Gehring, G. HÄllström (1963): A distortion theorem for functions univalent in an annulus. Ann. Acad. Sci. Fenn. Ser. A I, 325:1-16.

[HaL] G. H. HARDY, J. E. LiTTLEwoon (1932): Some properties of fractional integrals, II. Math. Z., 34:403-439.

[He] P. Henrici (1986): Applied and Computational Complex Analysis, vol. III. New York: Wiley.

[Hi] E. HILle (1962): Analytic Function Theory, vol. II. Boston: Ginn.

[HoT] L. H. Howell, L. N. TREFETHEN (1990): A modified Schwarz-Christoffel transformation for elongated regions. SIAM J. Sci. Statist. Comput., 11:928-949.

[Hu] F. HUCKEMANN (1962): Über einige Extremalprobleme bei konformer Abbildung eines Kreisringes. Math. Z, 80:200-208.

[La] R. LAUGESEN (1993): Extremal problems involving logarithmic and Green capacity. Duke Math. J., 70:445-480.

[Le] F. D. LeSLEY (1985): Conformal mappings of domains satisfying a wedge condition. Proc. Amer. Math. Soc., 93:483-488.

[LeV] O. Lehto, K. I. Virtanen (1973): Quasiconformal Mappings in the Plane, 2nd edn. Berlin: Springer-Verlag. Translated from the German by K. W. Lucas.

[Me] K. MENKE (1987): Distortion theorems for functions schlicht in an annulus. J. Reine Angew. Math., 375/376:346-361.

[MeZ] R. MENIKOFF, C. ZEMACH (1980): Methods for numerical conformal mapping. J. Comput. Phys., 36:366 410 .

[MoS] C. D. MOBLEY, R. J. STEWART (1980): On the numerical generation of boundary-fitted orthogonal curvilinear coordinate systems. J. Comput. Phys., 34:124-135.

[NäP] R. NÄKKI, B. PALKA (1986): Extremal length and Hölder continuity of conformal mappings. Comment. Math. Helv., 61:389-414.

[Pa] N. PAPAmichael (1989): Numerical conformal mapping onto a rectangle with applications to the solution of Laplacian problems. J. Comput. Appl. Math., 28:63-83.

[PaKW] N. Papamichael, C. A. Kokkinos, M. K. Warby (1987): Numerical techniques for conformal mapping onto a rectangle. J. Comput. Appl. Math., 20:349-358. 
[PaS1] N. Papamichael, N. S. STYlianopoulos (1990): On the numerical performance of a domain decomposition method for conformal mapping. In: Computational Methods and Function Theory (St. Ruscheweyh, E. B. Saff, L. C. Salinas, R. S. Varga, eds.). Lecture Notes in Mathematics, vol. 1435. Berlin: Springer-Verlag, pp. 155-169.

[PaS2] N. Papamichael, N. S. Stylianopoulos (1991): A domain decomposition method for conformal mapping onto a rectangle. Constr. Approx., 7:349-379.

[PaS3] N. Papamichael, N. S. Stylianopoulos (1992): A domain decomposition method for approximating the conformal modules of long quadrilaterals. Numer. Math., 62:213-234.

[PaS4] N. PAPAMICHAEL, N. S. StYlianopoulos (1993): On the theory and application of a domain decomposition method for computing conformal modules. J. Comput. Appl. Math., to appear.

[Po1] CH. Pommerenke (1975): Univalent Functions. Göttingen: Vandenhoeck \& Ruprecht.

[Po2] CH. Pommerenke (1992): Boundary Behaviour of Conformal Maps. Berlin: SpringerVerlag.

[SeK] A. SEIDL, H. KLOSE (1985): Numerical conformal mapping of a towel-shaped region onto a rectangle. SIAM J. Sci. Statist. Comput., 6:833-842.

[SmS] W. Smith, D. A. Stegenga (1987): A gèomètric charactétization of Hölder domàins. J. London Math. Soc. (2), 35:471-480.

[We1] R. WEGMANN (1992): An estimate for crowding in conformal mapping to elongated regions. Complex Variables Theory Appl., 18:193-199.

[We2] R. WEGMANN (1994): Crowding for analytic functions with elongated range. Constr. Approx., 10:179-186.

R. Laugesen ${ }^{1}$

Washington University

St. Louis

Missouri 63130-4899

U.S.A.

\footnotetext{
${ }^{1}$ Current address: University of Michigan, Ann Arbor, Michigan 48109-1003, U.S.A.
} 